\title{
Periodic-Pattern-selective Cells in Monkey Visual Cortex
}

\author{
Rüdiger von der Heydt, Esther Peterhans, and Max R. Dürsteler \\ Department of Neurology, University Hospital Zurich, $\mathrm{CH}-8091$ Zurich, Switzerland
}

To study the visual processing of periodic and aperiodic patterns, we have analyzed neuronal responses in areas $V 1$ and $\mathrm{V} 2$ of the visual cortex of alert monkeys during behaviorally induced fixation of gaze. Receptive field eccentricities ranged between $0.5^{\circ}$ and $4^{\circ}$.

We found cells that responded vigorously to gratings, but weakly or not at all to bars and edges. In some cells the aperiodic stimuli even reduced the activity below the spontaneous level. The distribution of a bar-grating response index indicated a discrete population of "grating cells" characterized by more than 10 -fold superiority of gratings. We estimated that these cells have a frequency of $4 \%$ in $\mathrm{V} 1$ and $1.6 \%$ in $V_{2}$, and that about 4 million grating cells of $V_{1}$ subserve the central $4^{\circ}$ of vision. The converse, cells that responded to isolated bars but not to gratings of any periodicity, was also observed. The grating cells of V1 were mostly (23 of 26$)$ found in layers 2,3 , and 4B. They preferred spatial frequencies between 2.6 and 19 cycles/degree (median, 9.3), with tuning widths at half-amplitude between 0.4 and 1.4 octaves (median, 1.0). Their tunings were narrower, and their preferred frequencies higher, than those of other cells on average. Grating cells were also narrowly tuned for orientation. Those of $\mathbf{V} 2$ were similarly selective. The responses of grating cells depended critically on the number of cycles of the gratings. With square waves of optimum periodicity responses required a minimum of 2-6 grating cycles and leveled off at 4-14 (median, 7.5). The corresponding receptive field widths were $0.34-2.4^{\circ}$ (median, $0.78^{\circ}$ ) for $V_{1}$ and $0.72-2.4^{\circ}$ (median, $1^{\circ}$ ) for V2. Grating cells typically gave unmodulated responses to drifting gratings, were unselective for direction of motion, and were strongly activated also by stationary gratings. Half of those of $\mathrm{V} 1$ were monocular, the others binocular, some showing strong binocular facilitation and disparity sensitivity. Length summation was usually monotonic, but strong end-inhibition was also observed. In contrast to other cells, grating cells were not activated by harmonic components. Spatial-frequency response curves for sine-wave, square-wave, and line gratings were similar. Square-wave gratings of one-third the preferred frequency failed to excite the cells, while the isolated $3 f$ component ( $f=$ the fundamental of the square wave) of these gratings evoked strong responses. In spite of the nonlinear features, grating cells had low contrast thresholds.

Received June 28, 1991; revised Nov. 11, 1991; accepted Nov. 12, 1991.

This work was supported by Swiss N.F. Grant 3.939.84. We thank Vappu Furrer-Isoviita for assistance in surgical operations and animal care.

Correspondence should be addressed to Dr. R. von der Heydt, Neurologische Universitätsklinik, Frauenklinikstrasse 26, CH-8091 Zürich, Switzerland.

Copyright (c) 1992 Society for Neuroscience $0270-6474 / 92 / 121416-19 \$ 05.00 / 0$
The detection performance of individual cells reached the performance of monkeys known from behavloral studles.

Since grating cells were not activated by the proper spatial-frequency components of edges and bars, and failed to signal the higher harmonics of gratings, which are essential for perception, we conclude that these cells do not serve a spatial-frequency analysis of the stimulus. They seem to be specialized for the detection of periodic patterns and may play a role in perception of texture. Responses to checkerboard patterns and to gratings with jittered periodicity supported this assumption. We argue that some studies designed to investigate spatial-frequency channels in human vision may have tested the performance of grating cells.

It is known that neurons in the visual cortex often respond better to gratings than to single bars (Glezer et al., 1973; Albrecht et al., 1980), but there have been no doubts that these neurons can be driven by bars and edges. The distinction between simple and complex cells, for example, is usually based on the spatial separation, or overlap, of excitatory and inhibitory subregions revealed by stimulation with bars and edges (Hubel and Wiesel, 1962, 1968). Several studies have used both bars and gratings to analyze the receptive fields and emphasized that both yield comparable descriptions (see von der Heydt, 1987, for review). For simple cells, it was generally concluded that the responses to bars and other stimuli can largely be predicted from the cells' spatial-frequency tuning (Movshon et al., 1978a; Andrews and Pollen, 1979; Maffei et al., 1979; Glezer et al., 1980; Kulikowski and Bishop, 1981), suggesting linear spatial filtering. Complex cells have been modelled by three-stage mechanisms such as linear filtering, rectification, and summation (Movshon et al., 1978b; Spitzer and Hochstein, 1985). Such complex cells again respond to bars as well as to gratings (Movshon et al., 1978b). Exclusive responses to gratings have not been described.

The question of linearity and the spatial frequency filtering properties of cortical neurons are of great theoretical interest. Many psychophysical studies have investigated visual perception in terms of the visibility of gratings (Campbell and Robson, 1968; for recent reviews, see Julesz and Schumer, 1981; Robson, 1983; Kelly and Burbeck, 1984; Westheimer, 1984; Graham, 1985, 1989; Shapley and Lennie, 1985; Olzak and Thomas, 1986; De Valois and De Valois, 1988; Wilson et al., 1989). The idea of an early stage of linear, spatial-frequency-selective channels underlying the perception of gratings as well as aperiodic patterns is widely accepted. Nonlinear processing is usually thought to occur at subsequent stages where the outputs of these channels are combined and perceptual decisions are made, for example, the decision if one of the channels was activated (Graham, 1977). On the other hand, severe physiological studies have demonstrated interactions between the channels such as 
"cross-orientation inhibition" (Morrone et al., 1982) and "crossfrequency inhibition" (De Valois and Tootell, 1983). These may have been regarded as minor modifications to the linear filter scheme.

In experiments with alert monkeys, recording from striate and prestriate cortex, we occasionally encountered cells that could not be driven by bars and edges but responded vigorously to gratings of appropriate periodicity and orientation. We were struck by this specificity, which seemed to contradict present concepts of neuronal function, particularly of the striate cortex. In the present study, we define these cells by a quantitative criterion, estimate their frequencies in $\mathrm{V} 1$ and in $\mathrm{V} 2$, and describe their responses. "Grating cells," as we call them, occur mostly in the superficial layers of $\mathrm{V} 1$ and form a relatively homogeneous group concerning various response properties. We show that these cells do not conform to linear filter or summation to threshold models; they fail to respond to the higher harmonics of gratings and the proper Fourier components of bars and edges, even though the equivalent gratings in isolation are very effective, but they seem to require periodic patterns of the proper periodicity. Thus, they seem to serve a more specialized function than spatial frequency filtering. On the other hand, a grating cell may respond to a variety of one-dimensional and two-dimensional periodic patterns. Some are very sensitive, responding at contrasts down to the psychophysical threshold.

These findings pose the question of whether grating cells exist also in the human visual cortex and play a role in perception. The notion of grating cells might lead to a new interpretation of the results of many psychophysical experiments on perception of gratings of the last 20 years and might resolve some old controversies. In contrast to the widely accepted theory of visual form processing assuming a stage of spatial-frequency-selective linear filtering plus subsequent nonlinear stages, the existence of grating cells in V1 seems to support an alternative view, namely, that various aspects of the image are processed in parallel by different families of cells and that some aspects are extracted by specific nonlinear mechanisms at an early stage.

We present here the main results of our experiments on grating cells. Our aim is to describe the stimulus conditions under which these cells are activated and thus to illuminate their function in vision. The possible mechanism underlying the responses of grating cells will be discussed and modeled in a forthcoming report (Mechanisms of periodic pattern selectivity in monkey visual cortex, R. von der Heydt, E. Peterhans, and M. R. Dürsteler, in preparation).

\section{Materials and Methods}

General. Monkeys (Macaca mulatta) were trained to fixate their gaze on a small target in the center of a stimulus display field. Training and behavioral control during recording sessions were achieved by mild liquid deprivation and rewarding (von der Heydt and Peterhans, 1989). The animals learned to detect a $90^{\circ}$ turn of a small target (two parallel lines) that could be resolved only in foveal vision. The change occurred after a variable "fore period" of $0.3-5 \mathrm{sec}$. Visual control on a TV monitor showing the eyes in magnification, and a high rate of correct responses indicated reliable fixation during this interval. The animals were prepared for semichronic recording under general anesthesia and aseptic conditions. Anesthesia was initiated by intramuscular injection of $5-10 \mathrm{mg} / \mathrm{kg}$ ketamine hydrochloride and subcutaneous injection of $0.05-0.1 \mathrm{mg} / \mathrm{kg}$ atropinum sulfuricum, and continued by intraperitoneal injection of $30 \mathrm{mg} / \mathrm{kg}$ sodium pentobarbital (Nembutal) and further doses as necessary.

Recording. During the recording sessions, the animal's head was fixed by means of a steel bolt implanted on the skull. Single units were re- corded with glass-coated platinum-iridium microelectrodes inserted through the dura, usually for one penetration per day. Sharply tapered, selective electrodes were used that also picked up some background activity (taper, 0.07-0.09; impedance, 3-5 $\mathrm{M} \Omega$ at $1 \mathrm{kHz}$ ). With these electrodes, 22 cells could be discriminated on average in vertical penetrations through V1, and fiber spikes were sometimes picked up in the white matter. Only well-isolated cell activity was analyzed. Advancing the electrode, we carefully monitored the entry into the cortex, the amount of single- and multiunit activity and its stimulus preferences for orientation and ocularity, the entry into the white matter, and so on. The corresponding depths were recorded graphically. Comparison of such track charts with the histological reconstructions showed that layers $4 \mathrm{~B}, 4 \mathrm{C}$, and 6 in $\mathrm{V} 1$ could often be identified, the entrance into layer $4 \mathrm{~B}$ by a drop in unresolved activity and a low density of isolatable units, layer $4 \mathrm{C}$ by its unresolved, monocularly driven, and non-orientation-selective activity (Poggio et al., 1977), and layer 6 again by a higher density of isolatable units.

Histology. The penetrations of the last week of recording were marked by electrolytic lesions. The area of recording was finally marked with four to eight sharply pointed, 0.25 -mm-thick tungsten pins, inserted, under ketamine anesthesia, using the electrode-positioning device. The animal was then deeply anesthetized and the brain perfused with Ringer's solution and $4 \%$ formaldehyde. The marked blocks of tissue were either embedded in celloidin or frozen, for cutting, or cut on the vibratome. The slices were stained with cresyl violet or thionine. The factor of shrinkage was determined from the distances of the marker pins, which allowed an accurate localization of the electrode tracks. The layers were designated according to Lund (1973) and Lund et al. (1981).

Visual stimulation. Stimuli and fixation target were generated electronically on the face of an oscilloscope and viewed by the monkey stereoscopically through an optical system; they appeared superimposed on a $42^{\circ} \times 30^{\circ}$ homogeneous background field, at a distance of $40 \mathrm{~cm}$. Bars and gratings were generated by modulating or blanking the intensity of a pair of rectangular rasters of 240 lines. The refresh rate was 100 $\mathrm{Hz}$. The rasters could be positioned and rotated, and their size adjusted according to the size of the receptive fields. Squares of $4^{\circ}$ or $2^{\circ}$ size were typically used. The oscilloscope was specially designed for high resolution and equipped with a yellowish green phosphor of short persistence (Ferranti A5, peak at $555 \mathrm{~nm}$ ). Its voltage-luminance characteristic was linearized over a $3 \mathrm{log}$ unit range. The contrast transfer function of the entire system was measured with a direct-view photometer equipped with a narrow slit aperture (PR-1980A Spectra Pritchard) and corrected for the modulation transfer function of the photometer. It was practically flat up to $20 \mathrm{cycles} /$ degree (c/deg), dropping to $50 \%$ at $35 \mathrm{c} / \mathrm{deg}$. The background luminance $\left(L_{0}\right)$ was $10 \mathrm{~cd} / \mathrm{m}^{2}$ or, in recent experiments, 36 $\mathrm{cd} / \mathrm{m}^{2}$ (tungsten filament, $6 \mathrm{~V}, 15 \mathrm{~W}$ ). For comparing bar and grating responses, square-wave gratings and bars with $L_{\max }=2 L_{0}$ and $L_{\min }=$ $L_{0}$ were used, resulting in a Michelson contrast of 0.33 , but the increment luminance was also varied to produce other contrasts. Sinusoidal and square-wave gratings of various modulations and constant mean luminance $2 L_{0}$ were used for assessing the effects of harmonic components.

Each receptive field was first studied carefully with various stimuli by listening to the responses. For recording, the stimuli were generally moved back and forth with constant speed, and sets of different patterns (e.g., light and dark bars, grating, and blank field) or parameter values were tested in pseudorandom order. Each pattern was presented for several fixation periods, until eight motion cycles were completed during fixation. The entire set was usually repeated three times. Several of these interlaced series were run in each cell, and the critical observations were confirmed by later repetitions, often after hours. For further details of our method of analyzing receptive fields and recording responses, see von der Heydt and Peterhans (1989).

Analysis of responses versus number of grating cycles. As discussed below, it is of interest to relate spatial-frequency tuning and receptive field size of cortical cells (De Valois et al., 1985). In order to determine the extent of spatial summation, we measured the responses to gratings of limited extent as a function of the number of grating cycles. In the theoretical case of a linear filter with Gabor function profile, the resulting summation curve would have a simple shape with two linear asymptotes describing rising slope and saturation (see Appendix). If, in addition, the output of the filter is passed through a threshold nonlinearity, as is frequently assumed for cortical cells, the summation curve starts with a horizontal segment up to the threshold size of grating and then rises. We use this quasi-linear model as a baseline for discussing our results. In order to estimate the thresholds, slopes, and saturation levels of the 


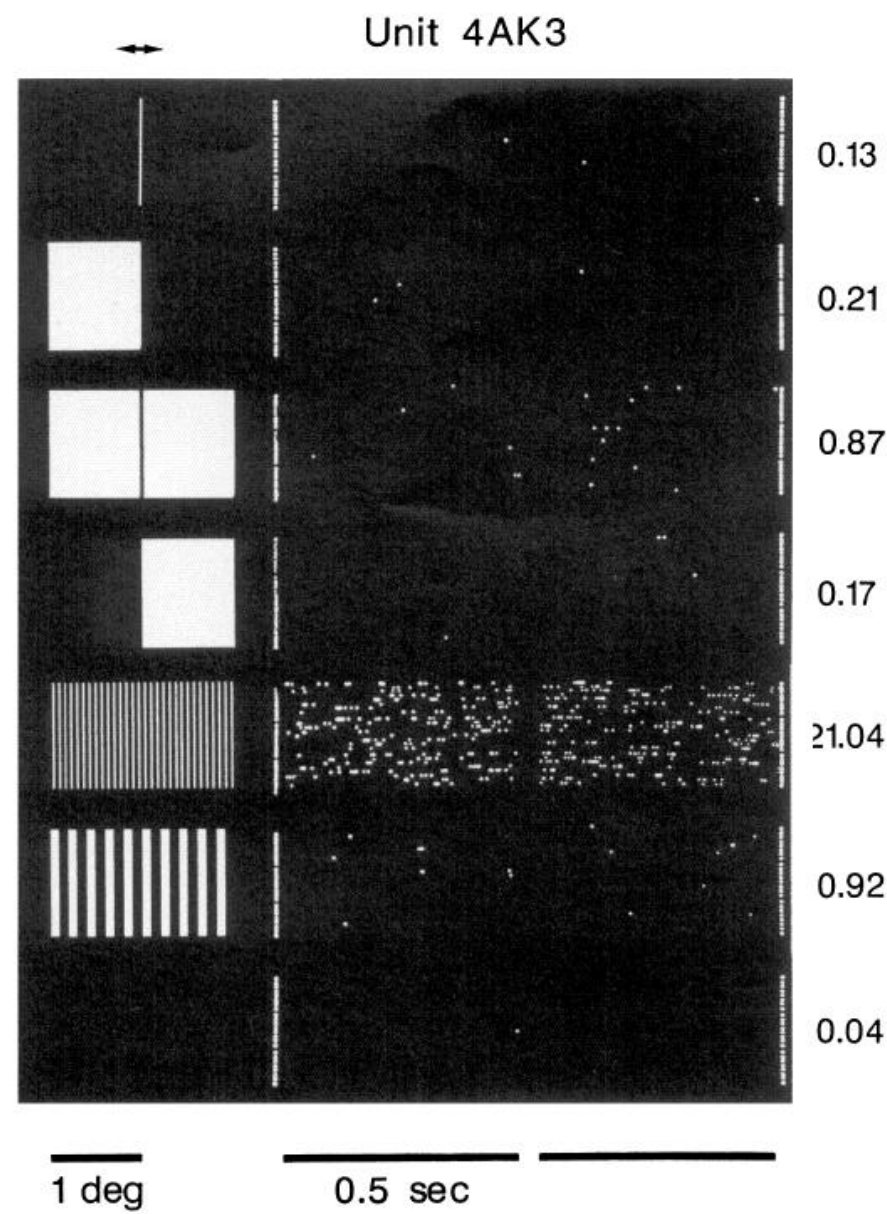

Figure 1. Pattern selectivity of a "grating cell" recorded in layers 2 and 3 of V1. Bars, edges, and gratings, as shown to the far left, were centered on the receptive field and oscillated back and forth. Bottom field, spontaneous activity. Each pattern was presented 24 times. The responses are shown between the white markers on the right, where each dot represents an action potential. Forward sweeps of movement are represented to the left, backward sweeps to the right, with reversed time axis. Mean numbers of spikes per presentation are shown to the far right. Typical for this type of cell was a vigorous response to gratings of some preferred periodicity and very weak responses or none at all to edges and single bars. Also, square-wave gratings of three times the preferred periodicity produced no response. Binocular stimulation with zero disparity; movement, $30 \mathrm{~min}$ arc, $1 \mathrm{~Hz}$. Bars: $2 \mathrm{~min}$ arc by $1^{\circ}$; gratings: 15 and $5 \mathrm{c} / \mathrm{deg}$ square wave, $4^{\circ}$ wide and $1^{\circ} \mathrm{long}$, orientation $14^{\circ}$ clockwise from horizontal (in the figure shown vertical for simplicity). Incremental luminance, $10 \mathrm{~cd} / \mathrm{m}^{2} ;$ background, luminance, $10 \mathrm{~cd} /$ $\mathrm{m}^{2}$.

cells accordingly, we have developed a method of "multiple phase regression." The algorithm determines the optimal way in the least-square sense of fitting the data points by $N$ segments of straight lines. Neighboring segments were made to share one data point, and the first segment, beginning with the point representing spontaneous activity, was forced horizontal. Fits for increasing numbers $N$ were calculated in sequence. $F$ statistics were calculated for comparison between residual variance and mean experimental variance (determined from repeated presentations), and between the residual variances of successive fits. The fit that reduced the residual variance significantly, compared to the preceding one, and brought it close to the mean variance of the data points was accepted as a description of the data. Two numbers were obtained from this fit, the "threshold," defined by the termination of the first (horizontal) segment, and the "point of saturation," defined by the beginning of the last (horizontal or descending) segment. We have done the same calculation also after a square-root transformation of the response values that equalized the variances that are normally higher for the greater responses, with only slightly different results.

\section{Results}

This study is based on the analysis of the responses to periodic and aperiodic stimuli of 130 cells of the visual cortex recorded in 91 electrode penetrations in four monkeys: 80 cells of area $\mathrm{V} 1,48$ of area V2, and 2 located near the border between these areas $( \pm 0.5 \mathrm{~mm})$. These cells were selected for analysis, as explained below, from a sample of 1446 cells recorded in 250 penetrations, 600 cells of $\mathrm{V} 1,749$ of V2, and 97 of the border region.

\section{Grating cells: definition and localization}

Figure 1 shows a typical example of the responses of what we call a "grating cell" (the precise definition will be given below). The cell was recorded in layers 2 and 3 of V1, and its receptive field centered at $1.4^{\circ}$ eccentricity. Responses to light and dark bars, edges, a square-wave grating of the optimum periodicity $(15 \mathrm{c} / \mathrm{deg})$, and a grating three times coarser are illustrated. The bottom field shows a comparable sample of spontaneous activity during fixation. The stimuli were centered on the most sensitive part of the receptive field, which was determined with a $2^{\circ} \times$ $2^{\circ}$ grating of optimum periodicity in the manner response fields are usually determined with bars. Mean firing rates are indicated on the right.

While the optimum frequency grating evoked 21 spikes per motion cycle, none of the other stimuli produced more than 1 spike per cycle. Responses were selective for the spatial frequency of square-wave gratings with a tuning width at halfamplitude of 1.0 octaves, and for the orientation of the grating with a tuning width of $21^{\circ}$. The other patterns failed to evoke responses also in other orientations. Many other stimuli, including bars of various lengths and stereoscopic stimuli with varied disparity, all gave negative results.

The absence of bar and edge responses appeared to be an important feature of such "grating cells" besides their selectivity for periodicity and orientation. We have therefore tried to classify the cells by the relative strengths of bar and grating responses. Out of 1446 cells whose receptive fields and orientation characteristics were determined, 1389 seemed to respond normally to bars or edges, while 57 gave unusually weak responses. In order to check our criterion, we have determined a bargrating response index for 96 cells, 39 with weak bar and edge responses, and 57 of the normal group for control. The latter were selected for recording before gratings were tested. The index was defined as

$$
\left(R_{\text {bar }}-R_{\text {grating }}\right) /\left(R_{\text {bar }}+R_{\text {grating }}\right),
$$

where $R_{\text {bar }}$ and $R_{\text {grating }}$ are the strengths of the bar and grating responses, respectively. Response strength was defined as the average number of spikes per movement cycle minus the spontaneous activity. For $R_{\text {bar }}$, the maximum of the light- and darkbar responses was used; for $R_{\text {grating }}$, the responses to gratings of the optimum spatial frequency. Since bar width was usually less critical than grating period, we set the bar width to half the cycle of the optimum grating in most cases. If critical, however, the optimum width was chosen. Bars and gratings were oscillated, usually at $1 \mathrm{~Hz}$, with an amplitude of two grating cycles, keeping the bars centered over the most sensitive part of the receptive field.

Figure 2 shows the distribution of the bar-grating response index. The stippled area under the histogram represents the cells recorded for their weak bar responses $(N=39)$; the open area, 
the control sample $(N=57)$. The rest of the large rectangle represents the other cells with apparently normal bar responses for which we do not have the bar-grating response index, assuming that they would be distributed over the same range. This is to show the approximate relative frequencies one would find in a random sample. The rectangular shape is of course arbitrary. Most of the other cells were examined quantitatively with aperiodic stimuli for other purposes. In general, the strong bar responses of these cells made it very unlikely that a grating response would be five times stronger, the factor represented by the left border of the rectangle. This was checked qualitatively by testing gratings of various frequencies in about one-third of the cells. Figure 2 includes cells of V1 (41\%), V2 (52\%), and the border region $(7 \%)$.

The distribution in Figure 2 shows a narrow peak near -1 , indicating a group of cells with virtually zero bar responses. Four cells had negative indices, which means that their activity was actually suppressed by bar stimulation compared to the spontaneous level. If we had taken the mean oif light- and darkbar responses instcad of their maximum, the index would be negative for seven cells. Suppression by bar stimulation was found consistently in each of these cells. Thus, the negative values do not just reflect variability in the determination of the index. In the following, we use the term "grating cells" for cells with a bar-grating response index below -0.825 (vertical broken line in Fig. 2), which means that the optimum-bar response was less than $10 \%$ of the grating response.

\section{Encounter frequencies}

Twenty-six (V1, 17; V2, 9) of the cells represented in Figure 2 met the criterion for "grating cells." In order to estimate the frequencies of grating cells in V1 and V2, we take into account also 13 cells with incomplete recordings that were judged to be grating cells during the examination by ear (strong grating response with virtually no response to bars), 9 of V1 and 4 of V2. Since of 34 complctely analyzed cells that were initially judged to be grating cells only $26(76 \%)$ actually met the quantitative criterion, we estimate that $0.76 \times 9=6.9$ of the $\mathrm{V} 1$ cells and $0.76 \times 4=3.1$ of the V2 cells with incomplete recordings had this property. This gives encounter frequencies of $23.9 / 600=$ $4.0 \%$ for $\mathrm{V} 1$, and $12.1 / 749=1.6 \%$ for $\mathrm{V} 2$. Of course, these estimates refer to the ensembles of cells as sampled by our electrodes (see Materials and Methods).

These figures may still underestimate the true frequency of grating cells because their stimulus selectivity and generally very low spontaneous activity reduce the chance of identifying such a cell. Their mostly superficial localization in striate cortex (see below) is another handicap because the preferred orientation of a column is not known at the beginning of a penetration, and the chance of holding a unit long enough immediately after penetrating the dura is lower than otherwise.

Laminar distribution. We noticed that grating cells in V1 were often encountered in the superficial layers. Four of them were the first cells isolated in a penetration. Of 26 grating cells of V1 19 were found in layers 2 and 3 , and 7 in the deeper layers ( 1 in $4 \mathrm{~A}, 4$ in $4 \mathrm{~B}, 1$ in $4 \mathrm{C}$, and 1 in layer 6), while of the other 574 cells, 284 were recorded in layers 2 and 3 and 290 in the deeper layers. (We have included the 9 presumed grating cells with incomplete recordings.) While the assignment of cells to single layers may be questioned, the tendency of grating cells to be found in the superficial layers was significant $\left(\chi^{2}=5.5 ; p<\right.$ 0.025 ). In area V2, 8 grating cells were found in layers 5 and

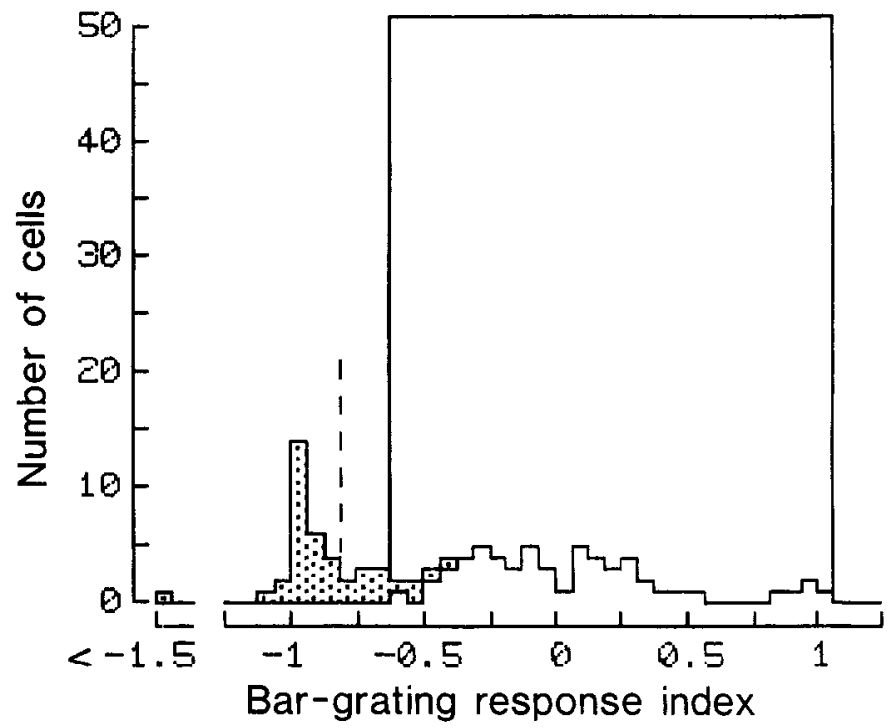

Figure 2. The definition of grating cells: distribution of the bar-grating response index (see Results, section 1) of 96 cortical neurons. Exclusive grating responses are characterized by an index of -1 ; exclusive bar responses, by an index of +1 ; and balanced preferences, inbetween. An index below -1 indicates that bar stimuli suppressed the activity below the spontaneous level. The stippled area of the histogram represents cells preselected for their apparently weak responses to bars and edges; the open area shows a representative control sample. The large rectangular area represents other cells with apparently normal bar responses. The broken line shows the criterion for the definition of grating cells.

6,5 in layer 4 , and none in 2 and 3 . About half of the other cells were recorded in layers 5 and 6 , and one-quarter each in layer 4 and layers 2 and 3 .

Absolute numbers. In order to estimate the absolute number of grating cells in the part of V1 that we have investigated, we use data on retinotopic mapping and cell density provided by anatomical studies. From Van Essen et al. (1984), their Figure 4 , we estimate that in macaque the central $4^{\circ}$ of visual field are represented by about $520 \mathrm{~mm}^{2}$ of striate cortex (V1). According to Rockel et al. (1980), a column of $20 \times 30 \mu \mathrm{m}$ of V 1 contains about 270 cells. However, the cell density is a factor of 2.5 higher in V1 than in other areas of neocortex, which is mainly due to the dense packing of cells in layer $4 \mathrm{C}$ (Rockel et al., 1980). Since grating cells were rare in layer $4 C$, and since cells in this layer might not be sampled adequately by the electrode, we base our estimate on the "normal" cell density of 110 per column, or $183,000 / \mathrm{mm}^{2}$, assuming that this is the density of the cells from which we have recorded. This gives $520 \times 183,000$ $=9.5 \times 10^{7}$ cells for the central part of V1. Since we estimate that $4 \%$ of these are grating cells, the monkey has a total of 3.8 $\times 10^{6}$ grating cells in V1 subserving the central $4^{\circ}$ of vision.

\section{Discrimination and summation properties}

\section{Spatial frequency tuning}

Many cells in V1 and V2 are selective for spatial frequency. Using the above definition, we can now compare grating cells with other cells. Since a simple monomodal spatial frequency dependence of the responses to square-wave gratings was one of the characteristics of grating cells (see section 3 below), we have generally used square-wave gratings for assessing spatial frequency selectivity. Therefore, our results on the control sample are not strictly comparable with the results of other studies 


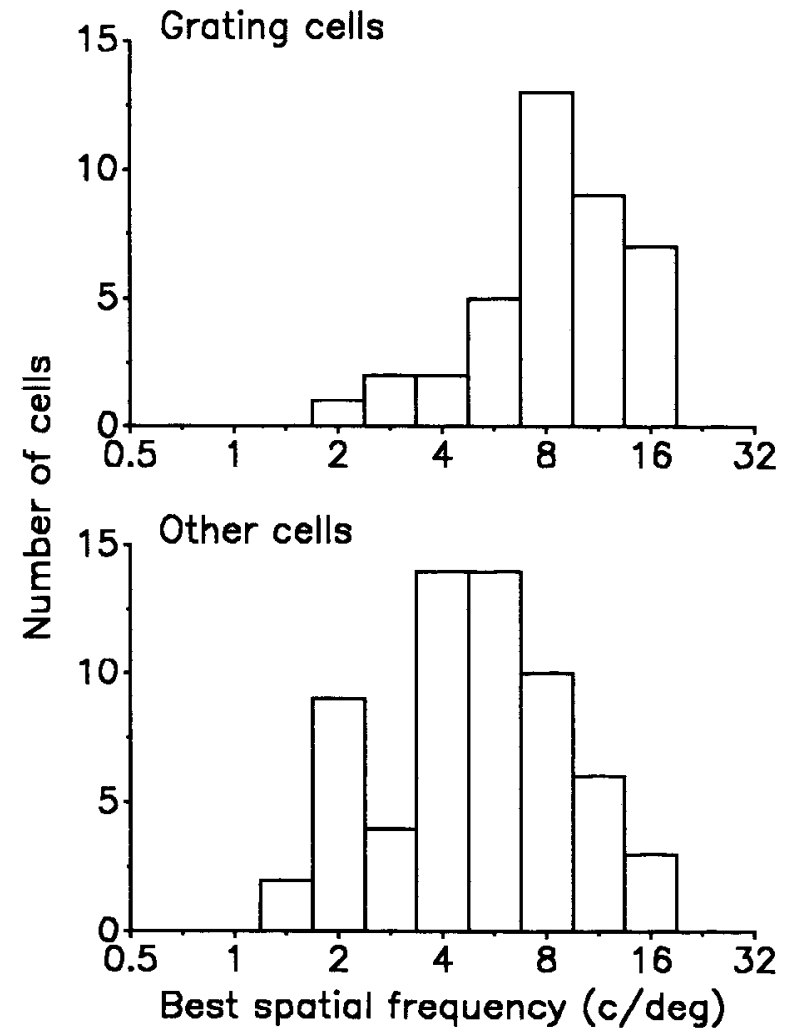

Figure 3. The distribution of the preferred spatial frequencies of grating cells $(n=39)$ and cells of the control sample $(n=62)$ determined with squarc-wavc gratings of about $33 \%$ contrast. Grating cells preferred higher frequencies on average. The figure includes cells from V1 (26 grating cells, 40 others) and V2 (13 and 22). For the exact comparison, see Results, section 2.

obtained with sinusoidal gratings, although the agreement is actually good (see Discussion).

Figure 3 shows the distribution of the peaks of tuning, for the grating cells and the "normal" cells; Figure 4 shows the corresponding distributions of the widths of tuning. The ratio in octaves between upper and lower cutoff frequencies, defined by a drop in response strength to half-maximum, was taken as the width, and the mean of the two cutoff frequencies was taken as the peak frequency. This mean was calculated on linear, logarithmic, or reciprocal frequency scales, depending on where the tuning curve was more symmetric (see below). We used this estimate of the peaks, rather than the spatial frequency of the highest measured response, because it is less sensitive to noise introduced by coarse sampling and the noise of the responses. We have also included the peak frequencies determined by ear of 16 grating cells and 17 other cells for which the tuning was not recorded. Excluded from the comparison sample were 5 cells that showed no low-frequency attenuation and 4 cells that did not respond to gratings at any spatial frequency (see below).

One can see that grating cells tended to have higher peak frequencies and narrower tuning than other cells. For Figures 3 and 4 the results of $V 1$ and V2 were combined. We have compared the two types of cells for V1 and V2 separately using the Wilcoxon-Mann-Whitney test. The median peak frequency in cycles per degree was 9.3 for the grating cells of V1 $(N=26)$

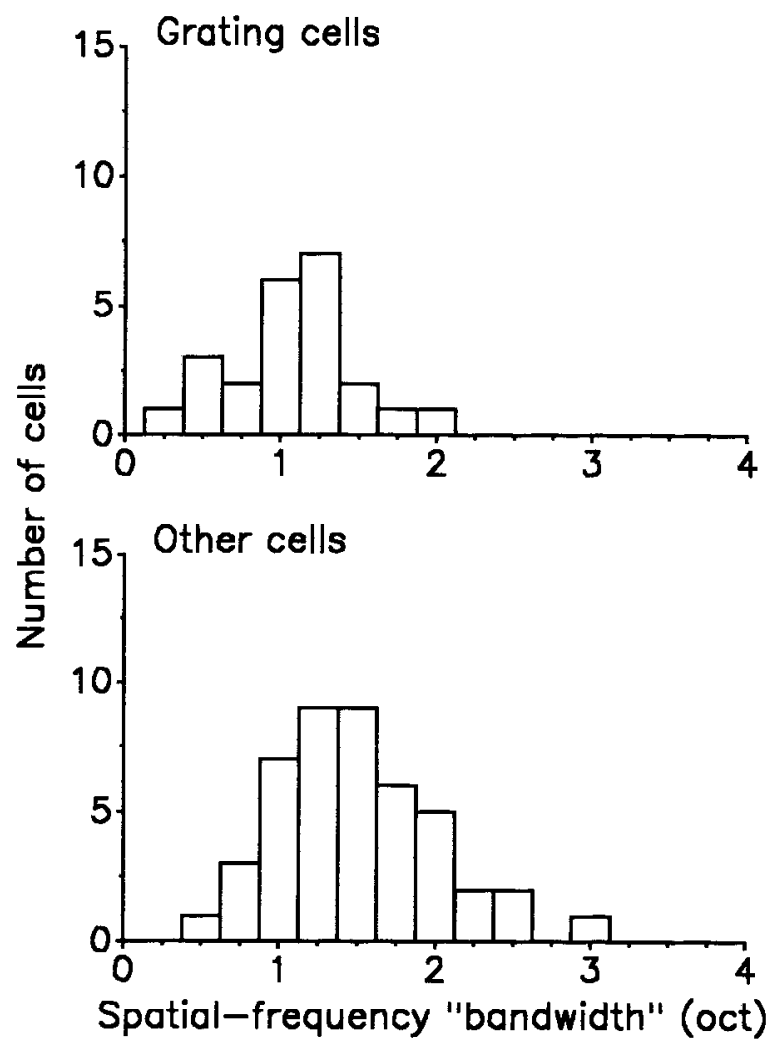

Figure 4. The distribution of the tuning widths of grating cells $(n=$ $23)$ and cells of the control sample $(n=45)$ as determined with squarewave gratings of about $33 \%$ contrast. Grating cells are more sharply tuned on average. The figure includes cells from V1 ( 15 grating cells, 31 others) and V2 (8 and 14). See Results, section 2, for the exact comparison. Note that these "bandwidths" do not describe the pattern selectivity of grating cells adequately because they are nonlinear (see Results, section 3 ).

and 6.0 for the other cells of V1 $(N=40)$, and 6.9 for the grating cells of V2 $(N=13)$ and 3.7 for the other cells of V2 $(N=22)$. For both areas the differences were significant at $p<0.005$. The median width of tuning in octaves was 1.00 for the grating cells of V $1(N=15)$ and 1.50 for the other cells of V1 $(N=31)$, and 1.35 for the grating cells of V2 $(N=8)$ and 1.49 for the other cells of V2 $(N=14)$. The width difference was significant at $p$ $<0.001$ for $\mathrm{V} 1$, but not significant for V2.

The comparison between areas $\mathrm{V} 1$ and $\mathrm{V} 2$ showed that the median peak frequencies of grating cells $(9.3$ and 6.9) were significantly different at $p<0.05$, those of the other cells (6.0 and 3.7 ) at $p<0.005$, while the median widths differed at $p<0.05$ for the grating cells (1.00 and 1.35), but not for the other cells.

The spatial-frequency and orientation tuning curves of a sharply tuned grating cell are shown in Figure 5 . Its preferred periodicity was $19 \mathrm{c} / \mathrm{deg}$; the widths at half-height were 0.43 octaves for spatial frequency and $12^{\circ}$ for orientation. The insets above each curve show the optimum grating and the gratings of half-maximal responses. During the recording we had been looking at a magnified replica of the stimulus on a slave scope, and when we saw the actual stimuli after the session we were surprised at the subtlety of these differences.

The spatial-frequency tuning of grating cells was not much affected by velocity of movement. The cells usually responded very well to stationary gratings and preferred the same frequency for moving and stationary gratings. The contrast had some effect 


\section{Unit 2DN2}
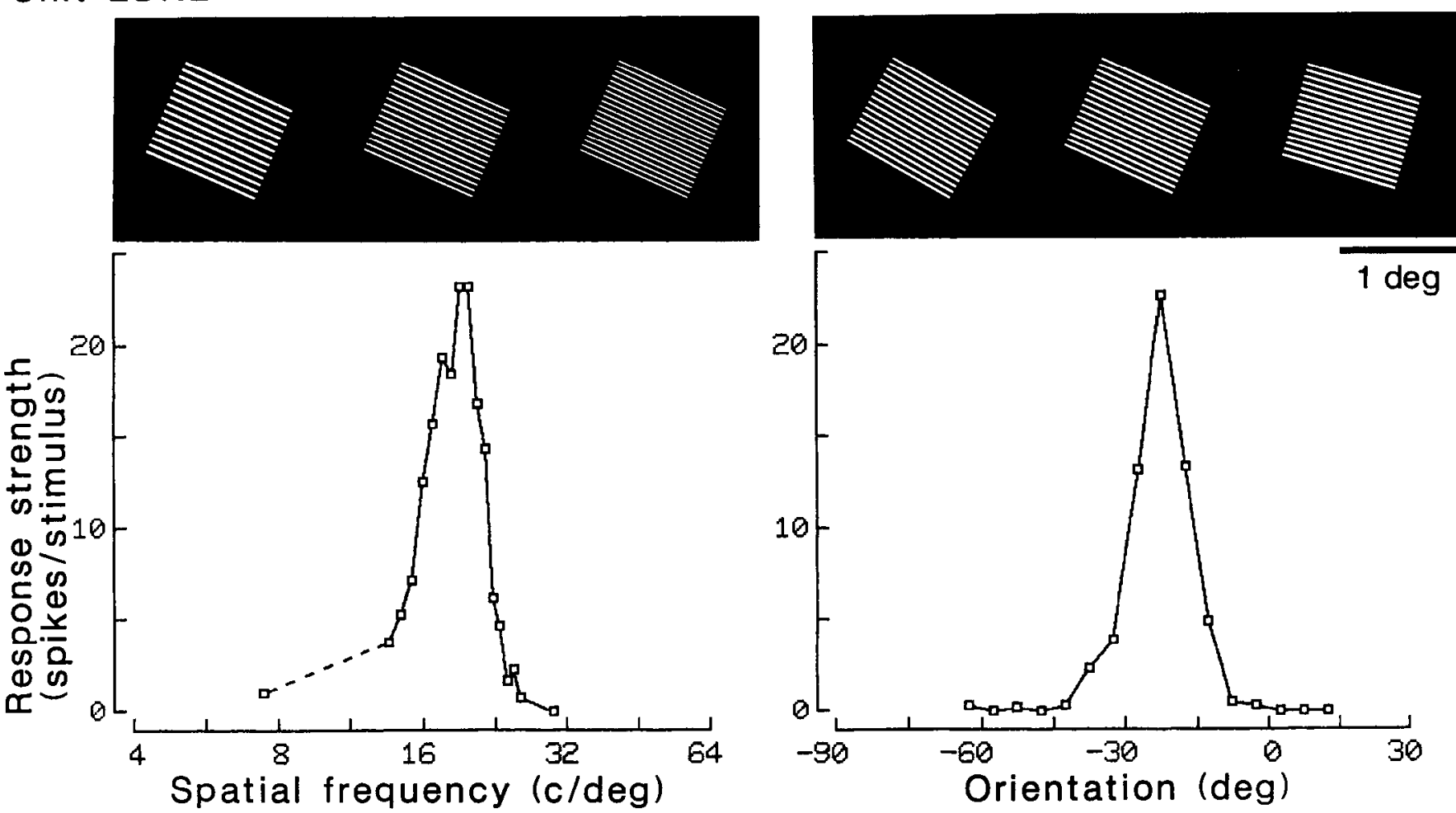

Figure 5. The tuning to spatial frequency and oricntation of one of the most sclective grating cells. The optimum grating and the gratings of halfmaximal responses are shown above the curves. To appreciate the cell's selectivity, the figure should be held at a distance of 57 times the calibration mark. The original gratings were square waves of 40 cycles and $60 \%$ contrast. Movement: 8 min arc, $1.5 \mathrm{~Hz}$. Monocular, contralateral stimulation.

on the width of tuning. Square-wave gratings of $62 \%$ contrast, and sinusoidal gratings of $55 \%$ and $18 \%$ contrast produced, respectively, widths of $1.17,1.00$, and 0.73 octaves in cell $3 \mathrm{BF} 1$, and $1.06,0.96$, and 0.85 in cell 4 CA4. We have not studied systematically the possible interaction between orientation and spatial frequency. However, it was clear that spatial frequencies that produced no response at the preferred orientation were ineffective also at other orientations, and, vice versa, orientations outside the responsive range at the preferred spatial frequency failed to evoke responses also at other frequencies.

Symmetry of tuning. We noticed that the tuning curves of most grating cells were fairly symmetric in the log-frequency plot but were left-skewed in linear plots. This was the case for 14 out of 23 cells (e.g., cells $3 \mathrm{BF} 1$ in Fig. 10 and $3 \mathrm{HK} 1$ in Fig. 12 below); for 2 cells the curves were most symmetric on the linear frequency scale (cell 2DN2 in Fig. 5); for 2 cells, on the linear cycle scale; and for 3 cells the issue was not clear. It is of interest here to note that the tuning of a linear filter with Gaborfunction profile is symmetric on a linear frequency scale but is right-skewed on a log-frequency scale. Thus, grating cells differ from Gabor filters in the shape of tuning.

Dependence of spatial-frequency tuning on eccentricity. Since our sample of cells represents receptive fields at various eccentricities between $0.5^{\circ}$ and $4^{\circ}$, some of the scatter seen in Figures 3 and 4 may be due to this variable. Analysis of regression showed that the logarithm of the peak frequencies decreased with eccentricity at a rate of -0.27 octaves/degree in $\mathrm{V} 1(t=$ $-2.45, \mathrm{df}=63, p<0.02$, two-sided test), whereas no trend was found in $\mathrm{V} 2(t=-0.14$, $\mathrm{df}=32, p>0.8)$. The slopes of regression were not different for grating cells and other cells (V 1: $t=0.67, \mathrm{df}=62, p>0.5)$. Thus, the two groups could be described by parallel regression lines vertically offset by 0.60 octaves in $\mathrm{V} 1(t=-3.30, \mathrm{df}=63, p<0.002)$ and 0.80 octaves in $\mathrm{V} 2(t=-2.35, \mathrm{df}=32, p<0.05)$. At zero eccentricity we found mean peak frequencies of $12.1 \mathrm{c} / \mathrm{deg}$ for the grating cells and $8.0 \mathrm{c} / \mathrm{deg}$ for the other cells in $\mathrm{V} 1$. The corresponding values for $\mathrm{V} 2$ are 6.1 and $3.5 \mathrm{c} / \mathrm{deg}$. In either area the regression accounted for only about $20 \%$ of the variance. The standard deviation of the residual scatter was 0.73 octaves in V1 and 0.79 octaves in V2. Thus, at any eccentricity, the peak frequencies cover a range (6 SDs) of about 4.5 octaves in each group of cells in each area. In contrast, the eccentricity caused a variation of mean pcak frequency of only onc octave in the range that we have explored. The widths of tuning did not change significantly with eccentricity (V1, 0.12 octaves/degree; V2, -0.22 octaves/ degree; $0.1<p<0.2$ in both cases).

\section{How many bars make a grating?}

When a cell responds to a grating, but not to a single bar of it, one wonders how such a cell would react to a grating of a few bars. How many cycles of grating are analyzed by a receptive field is of interest also if one considers the spatial-frequency tuning, since, for linear filters, there is an inverse relationship between the extent of sampling and the width of tuning (cf. Marcelja, 1980).

This experiment was carried out in 38 cells, 18 of which were grating cells. Gratings of optimum periodicity were used. The first bar was centered on the most sensitive point of the receptive ficld, and the further bars were added alternatingly on either side. The amplitude of movement was set to two grating cycles. 
Figure 6. The strength of responses as a function of the number of cycles of square-wave grating stimuli. $A$, Curves of five representative grating cells. $B$, Curves of four other cells for comparison. Horizontal broken lines indicate the levels of spontaneous activity. The grating cells showed a "threshold" for the number of bars between 1 and 5 , and summation up to $6-12$, while the other cells responded to a single bar, and addition of further bars could increase or decrease the response strength. Periodicities, from top to bottom: $A$, $10.6,7.5,4.3,15$, and $7.5 \mathrm{c} / \mathrm{deg} ; B, 3.8$, $7.5,4.3$, and $15 \mathrm{c} / \mathrm{deg}$. Bar-grating index, from top to bottom: $A,-0.90$, $-0.93,-0.86,-0.92$, and $-0.98 ; B$, $-0.28,-0.32,-0.03$, and +0.89 . Cell $3 H G 2$. in layer 6 of the $\mathrm{V} 1 / \mathrm{V} 2$ border region; the others, in layer $2 / 3$ of $\mathrm{V} 1$. $2 H L 2$ was recorded in layer 6 of $\mathrm{V} 2$;
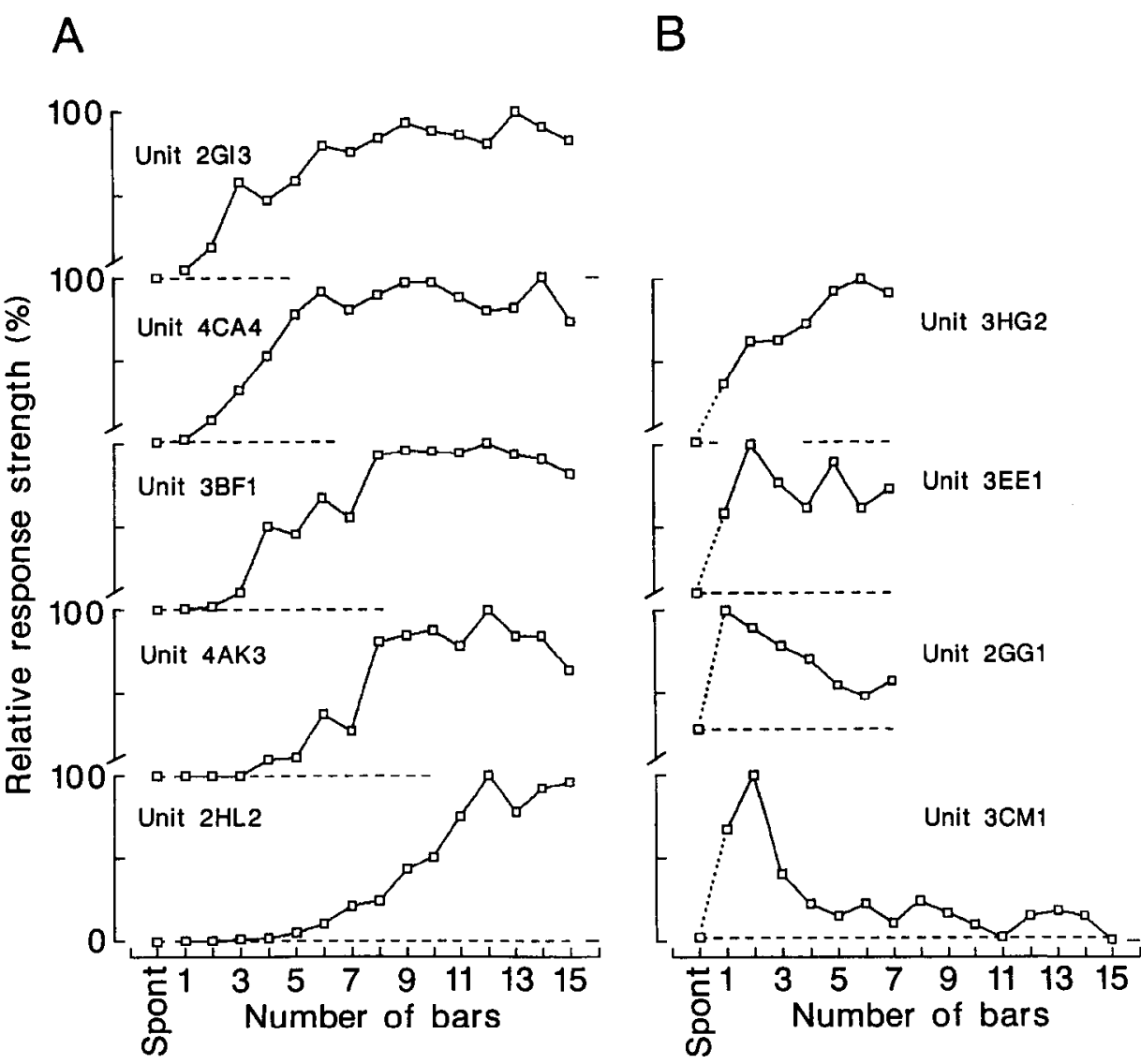

In Figure 6 the normalized response strength is plotted as a function of number of bars for five representative grating cells in $A$ and four other cells in $B$. The grating cells all showed the gradual increase of responses from zreo to a maximum that was reached only with many bars. Sometimes there was a slight decline beyond the maximum. The other cells showed either increasing or decreasing responsiveness, or even oscillations, when further bars were added. The four curves in Figure $6 B$ illustrate the variety of results that can be obtained. Three of these cells were band-pass tuned to spatial frequency, with bandwidths of $0.9,1.9$, and 1.2 octaves, respectively, from top to bottom, comparable to those of the grating cells. The bottom curve in $B$ is an example of a cell that responded to isolated bars but could not be driven by gratings of any periodicity. Such cells are characterized by bar-grating response indices near +1 (cf. Fig. 2). Cell 3CM1 responded selectively to narrow dark bars; the optimum width was 6 min arc. The best response to a grating was $1.4 \mathrm{spikes} / \mathrm{sec}$, obtained with a periodicity of 15 $\mathrm{c} / \mathrm{deg}$. The bottom curve in Figure $6 B$ shows that, when this grating was limited to various numbers of dark bars on a light field, the cell responded best with one or two bars and less when more bars were added.

The curves of the 18 grating cells were all of the same general shape, but the beginning and end of the rising slopes were different. We have determined these two points from each curve by finding the minimum combination of straight line segments, with a horizontal first segment, that provided an adequate fit of the data points (see Materials and Methods). In 15 cases this was three segments; in three cells, four or five segments were required. We denote the abscissa of the end of the first (hori- zontal) segment by $n_{0}$, and the abscissa of the beginning of the last segment, which was nearly horizontal or descending, by $n_{1}$. The value of $n_{0}$ ranged from 0.5 to 4.9 but was close to 1 in 10 of the 18 cells (median, 1.5); $n_{1}$ ranged from 4.0 to 13.6 (median, 7.5). Note that these are full grating cycles. Other studies have quantified spatial summation by numbers of half cycles, in analogy to the numbers of subregions in simple receptive fields. We point out also that our $n_{1}$ is not the number of cycles that produced the maximum response, but rather an estimate of the termination of the linear slope. The $n$ of maximum response was usually greater than $n_{1}$. To characterize the shape of the summation curves, we calculated also the ratio $n_{0}: n_{1}$. A ratio of 0 means a linear increase; a ratio of 1 , a steplike function. The grating cells had ratios between 0.08 and 0.50 (median, 0.23 ).

Control for movement amplitude. One could ask whether the movement of the gratings contributed to our estimate of $n_{1}$. The movement tends to smear the transition from the rising slope to the horizontal asymptote. Our procedure is relatively insensitive to this effect because it determines the intersection of lines that estimate the slope and the asymptote. For control we have recorded, for one cell, three summation curves with different amplitudes of motion: $14 \mathrm{~min}$ arc $(1$ cycle), $35 \mathrm{~min}$ arc $(2.5$ cycles), and $70 \mathrm{~min}$ arc $(5$ cycles). This resulted in estimates of $n_{1}$ of $8.5,9.0$, and 10.0 , respectively. Thus, the influence of the amplitude of movement was small. Since we used small amplitudes (two grating cycles) for the experiment of Figure 6, the effect of the movement can probably be neglected.

Spatial summation and frequency tuning. If we would carry out the experiment of Figure 6 on a Gaussian filter (linear filter with Gabor-function profile), we would obtain a summation 

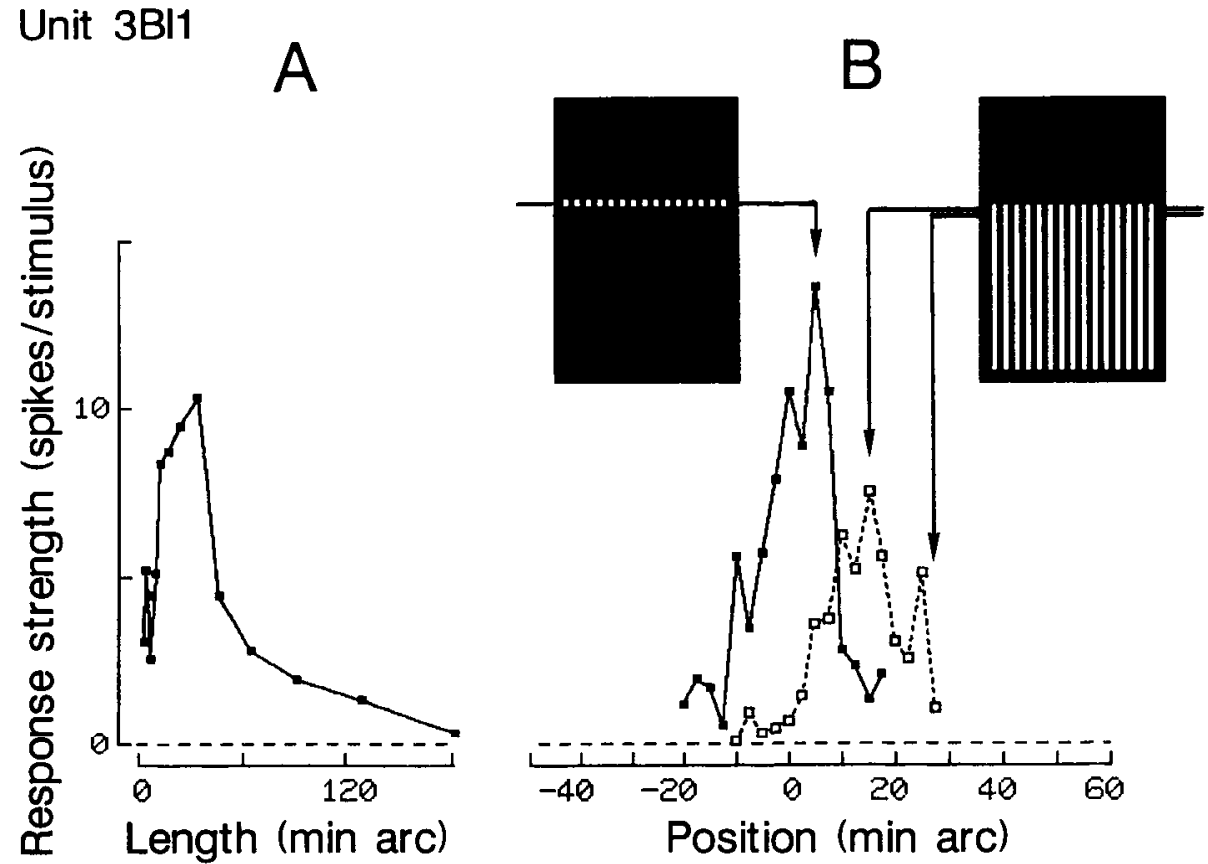

Figure 7. Example of an end-stopped grating cell. $A$, Effect of varying the length of grating symmetrically about position zero. Best responses were obtained with lengths of 10-30 min arc. $B$. Effect of varying vertical position. Solid curve, short grating (10 min arc; abscissa; position of center); broken curve, long grating ( $240 \mathrm{~min}$ arc; abscissa, position of edge). Horizontal lines in the stimulus insets show, for three of the data points, where the receptive field center was located relative to the gratings. The cell responded only within about 20 min arc of vertical position, little more than the range of fixational eye movements. Such cells may signal borders of texture. The cell was recorded in layers 2 and 3 of V1. Bar-grating index, -0.91 ; preferred periodicity, 3.4 c/deg; "bandwidth," 0.71 octaves; orientation, $5^{\circ}$ left of vertical; tuning width, $17^{\circ}$. Stimuli were drawn vertical for simplicity.

function that rises first linearly and then levels off to approach a horizontal asymptote (see Appendix). The intersection of the linear slope with the horizontal asymptote, expressed in number of grating cycles, corresponds to our $n_{1}$. This number is related to the spatial frequency bandwidth of the Gaussian filter as derived in the Appendix. It turns out that $n_{1}=7.5$, as found for the median grating cell, corresponds to a bandwidth of 0.18 octaves. This is much smaller than the median bandwidth of the grating cells, which was 1.0 octaves in V1 and even greater in V2, and is considerably less than even the smallest bandwidths that we have found ( 0.4 octaves; Fig. 4$)$. In other words, grating cells sum more cycles than would be necessary to achieve the tuning that they actually show. To quantify this we have calculated, for each cell, the ratio between the experimentally determined $n_{1}$ and the theoretical $n_{1}$ of a Gaussian filter with the same width of tuning. The ratio ranged between 2.2 and 11.2 , with a mean of $5.8(N=15)$. Thus, if one assumes that the receptive field of a grating cell is composed of subunits with receptive fields of the size of such Gaussian filters, the ratio between total size and subunit size ("complexity index"; Glezcr et al., 1980) is 5.8 on the average. This is not to say that the subunits actually resemble Gaussian filters, nor that they are linear filters (see below).

Receptive field size. The product of $n_{1}$ with the grating cycle is a measure of the receptive field width according to the definition of $n_{1}$. For the grating cells of V1, the width ranged between $0.34^{\circ}$ and $2.3^{\circ}$, with a median of $0.78^{\circ}(N=13)$; for those of $\mathrm{V} 2$, between $0.72^{\circ}$ and $2.4^{\circ}$, with a median of $1.41^{\circ}(N=5)$. Thus, although the cells integrate many cycles, they do not have very large receptive fields. The receptive field borders did not seem to be sharp. Using gratings of half the limiting size, for example, four bars, oscillating with small amplitude, we obtained position-response curves with gradual rise and decline, similar to Gaussian functions. As noted above, the summation curves of some cells showed a decline beyond the peak (cells 4AK 3 in Fig. 6 and 3BE2 in Fig. 16), suggesting surround inhibition.

\section{Length summation and end-stopping}

Most grating cells showed monotonic length summation, usually up to $1-2^{\circ}$. In these cells, positioning of the grating in the length direction was not critical. With a short grating, position-response functions were bell shaped, and when a border of a long grating was moved into the receptive field from either end, responses increased gradually. This means that the location of the grating borders was not accurately encoded.

However, a few grating cells, six of V1 and one of V2, showed strong end-inhibition so that they responded only weakly or not at all to gratings covering the entire receptive field. Lengths between 5 and $30 \mathrm{~min}$ arc were optimal in these cells when the grating was centered, but in two of them we found that one end of a long grating evoked nearly as good a response, and the position of the grating border was critical. Figure 7 illustrates the length summation and the effects of positioning a short grating and a grating border in a cell recorded in layer $2 / 3$ of V1. Displacements of the grating border of $10 \mathrm{~min}$ arc from the optimum position to cither side decreased the responses by $50 \%$. Similar behavior was found in a cell of V2 (cell $2 \mathrm{HL} 2$ in Fig. 6). These cells seem to be sensitive to borders of gratings. The feature of end-stopping adds a new twist to the specificity of the grating cells.

\section{Direction selectivity}

Most grating cells were insensitive to the direction of movement of the gratings. Of 29 cells, only one was completely direction selective and two were direction sensitive with a response strength ratio of $2: 1$. The completely selective and one of the partially selective cells were found in layer $4 \mathrm{~B}$ of $\mathrm{V} 1$; the other was found in layer 4 of $\mathrm{V} 2$. In the other 26 cells the responses to the two directions of motion did not differ more than 10:7. All these cells could be activated strongly by stationary, unmodulated gratings. Apparently the fixational eye movements are sufficient to maintain this activity. 


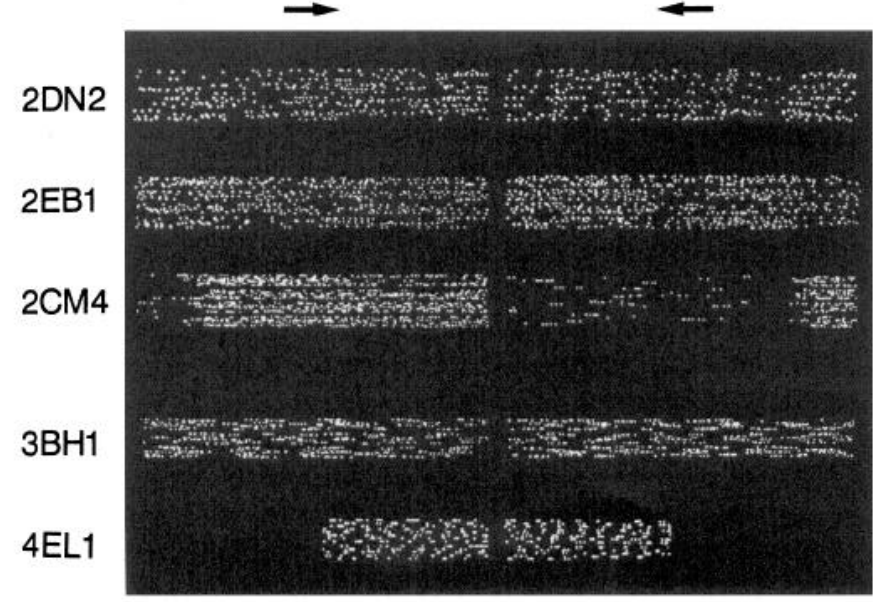

Figure 8. Dot displays of the responses to moving gratings of three grating cells; a simple cell recorded in V1, and an on-center lateral geniculate fiber recorded in the white matter below the striate cortex. The simple cell $3 B H I$ (spatial frequency, $10 \mathrm{c} / \mathrm{deg}$; movement amplitude, 6 cycles) and the LGN fiber $4 E L 1$ ( $15 \mathrm{c} / \mathrm{deg}, 8$ cycles) produced clearly modulated responses. This is visible in the displays in spite of variations in the phase of response due to fixational eye movements and high spatial frequency. Virtually no modulation was found in the responses of the grating cells ( $2 D N 2,20 \mathrm{c} / \mathrm{deg}, 3$ cycles; $2 E B 1,7.5 \mathrm{c} / \mathrm{deg}$, 2 cycles; $2 C M 4,7.5 \mathrm{c} / \mathrm{deg}, 2$ cycles). Cell $2 C M 4$ is a rare case of a direction selective grating cell. During drift in the preferred direction it responded with a mean firing rate as high as $130 \mathrm{~Hz}$. Representation as in Fig. 1; arrows indicate direction of time axis. Motion cycles were $0.66 \mathrm{sec}$ for cells $2 D N 2$ and $2 C M 4$, and $1 \mathrm{sec}$ for the others. Thirty-two repetitions are represented for the grating cells; 24 and 13 for units $3 B H 1$ and $4 E L 1$.

\section{Ocular dominance}

We noticed that grating cells in V1 were often driven only from one eye and showed no binocular interaction. Of 16 cells for which we have records on ocular dominance and binocular interaction (based on grating responses), 7 were of this type and 3 gave only weak responses from the nondominant eye; 4 could be driven about equally well from either eye, and 2 others responded weakly to monocular stimuli but showed strong binocular facilitation. These latter were sharply tuned for horizontal disparity with peaks near zero. Thus, nearly half (7 of 16) of the grating cells of V1 were purely monocular (contra, 3; ipsi, 4). In general, purely monocular cells are rare in the visual cortex, Poggio (1984) estimates 5-6\% in V1. To find such cells in the superficial layers is particularly unusual.

The ocular dominance of the grating cells in V2 was not unusual. Five of seven cells were driven about equally well from either eye; two responded only binocularly and were sharply tuned for horizontal disparity.

\section{Simple or complex?}

It is obviously impossible to classify grating cells as simple or complex by the classical criteria of Hubel and Wiesel (1962, 1968), since this would require bar responses. However, the modulation of responses to moving gratings could be used as a criterion (Maffei and Fiorentini, 1973; Ikeda and Wright, 1975; De Valois et al., 1982; Dean and Tolhurst, 1983). The distinction is less easy when recording from the alert monkey because residual eye movements change the phase of response so that one cannot determine the modulation from averaged responses. Nevertheless, Poggio et al. (1977) were able to distinguish "mod- ulated" and "fusional" cells, a distinction that is obviously related to the simple/complex classification.

Responses to drifting gratings. By listening to the responses to moving gratings, we found that most grating cells responded with unmodulated activity. We have also searched in the dot displays for patterns of modulation with the expected periodicity. In the responses of 28 grating cells, we found no indication of modulation in 18 cases; we were not sure in 7 cases and found clear modulation in 3 cases. Figure 8 shows three more examples of the cells with unmodulated responses (see also Fig. 1). On the other hand, we could clearly hear the modulation in the responses of simple cells and geniculate fibers at high spatial frequencies, for example, in neurons $3 \mathrm{BH} 1$, with $10 \mathrm{c} / \mathrm{deg}$, and 4EL1, with $15 \mathrm{c} / \mathrm{deg}$ (Fig. 8). The modulation is less visible in these displays than it was audible because its phase changes randomly between the lines due to variation in fixation of gaze (cf. Motter and Poggio, 1984). Thus, the majority of grating cells would be "complex" by this criterion.

\section{Spatial-frequency analysis or periodic pattern detection?}

Because of their selectivity for spatial frequency and orientation, one might think that grating cells serve to extract Fourier components from the stimulus. However, the problem with this idea is that all the stimuli shown in Figure 1 have spectral components within the pass-band of the cell illustrated, but except for the grating of the preferred frequency, none of them produced a response. For the light and dark bars, for example, one can easily calculate that about $30 \%$ of the spectral energy would pass a linear filter with a one octave pass-band (Fig. 9). Thus, the unresponsiveness to bars contradicts the spatial-frequency filter assumption. Similarly, the coarser square-wave grating of Figure 1 has a third harmonic at the cell's preferred frequency, which has $42 \%$ of the modulation of the grating, but again there were no responses to this grating. Clearly, the high-frequency components are important for the appearance of these stimuli; otherwise, all bars would look blurred, bars of different widths would only differ in brightness, and sine- and square-wave gratings would look the same. However, this cell apparently ignored these frequency components. It seemed to respond only when its preferred frequency matched the periodicity of the stimulus.

\section{Harmonics of gratings}

All grating cells showed simple, unimodal frequency tuning curves when tested with square-wave gratings, indicating that the harmonics had no effect. In Figure 10 we compare the tuning curves obtained with square-wave and sinusoidal gratings in two cells, a grating cell of layer $2 / 3(A)$ and a "normal" complex cell of layer $4(B)$. Both cells showed similar sine-wave tuning (si). With square waves $(s q)$, however, a secondary peak at onethird the optimum frequency was obtained in cell $B$, but not in cell $A$. The arrows indicate frequency and amplitude of the secondary peaks that would be predicted, on the assumption of linearity, from the cells' sine-wave tuning curves. The responses of cell $B$ conformed exactly to the prediction.

We wondered whether the absence of responses was due to the relatively low modulation of the third harmonic and a high threshold of the grating cell. In a square-wave signal of modulation $M$, the modulation of the third harmonic is $0.42 M$. In the experiment of Figure 10, for example, the square-wave gratings had a contrast of 0.6 , resulting in a modulation of the third harmonic of 0.25 . We have therefore recorded, in five grating 


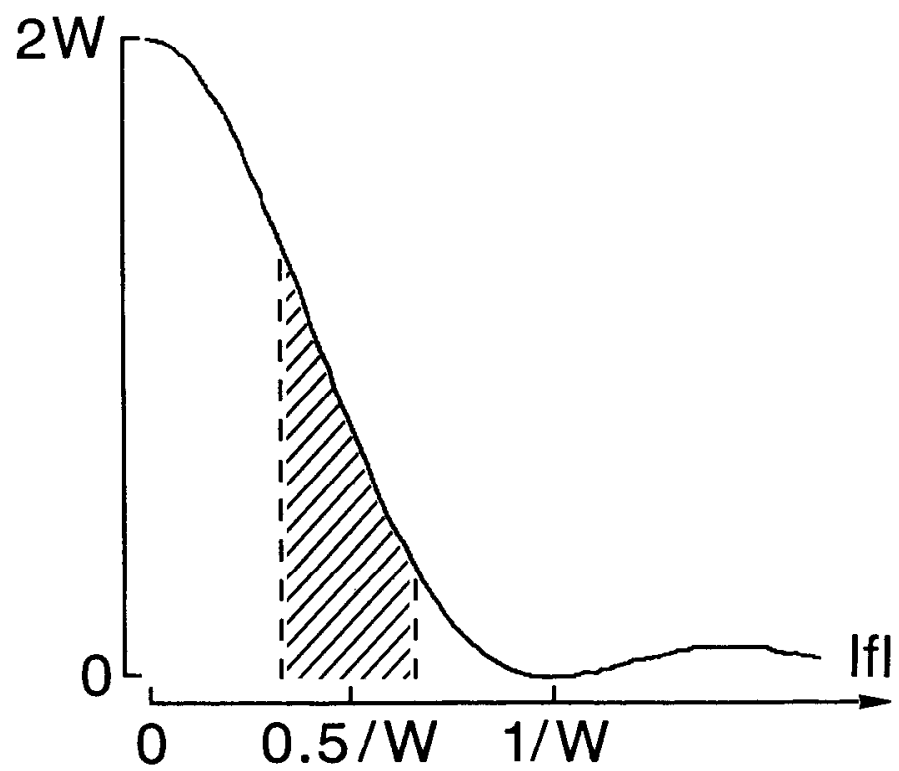

Figure 9. Although the Fourier spectrum of a single bar is quite different from that of a grating of alternating light and dark bars, it has a significant portion of spectral energy in the vicinity of the grating frequency. The figure illustrates this for a bar of width $W$ and a one-octave bandpass filter centered on the grating frequency $0.5 / W$. The curve shows the energy spectrum of the bar, $E(f)=2 W \cdot[\sin (\pi W f) / \pi W f]^{2}$, normalized for a total energy (area under the curve) of 1 . The hatched area equals the fraction that passes the filter (for simplicity, a rectangular filter characteristic is assumed). The cutoff frequencies are $1 / 3 W$ and $2 / 3 \mathrm{~W}$, and the width of the hatched strip is thus $1 / 3 \mathrm{~W}$. Its height at $f$ $=0.5 / \mathrm{W}$ equals $0.81 \mathrm{~W}$, as calculated from the above expression. Therefore, the hatched area equals approximately $0.81 \mathrm{~W} \cdot 1 / 3 \mathrm{~W}=0.27$. Thus, $27 \%$ of the energy passes the filter.

cells and a few other cells, the responses to square-wave gratings of one-third the optimum frequency and to the sine-wave gratings corresponding to their third harmonics. The luminance profiles of these stimuli are shown in Figure $11 A$; the results are plotted in Figure $11 B$. Each of the six cells represented was sufficiently narrowly tuned to the frequency $3 f(f=$ the fundamental of the square wave) so that sinusoidal gratings of frequency $f, 7 f$, and higher frequencies produced no responses and $5 f$ evoked only very weak responses at most (cf. the sine-wave tuning curves of Fig. 10). Thus, for linear filters of corresponding tuning, the two gratings would be nearly equivalent. However, they were not for the grating cells. These failed to respond to the full square waves (Fig. $11 B$, solid bars) even though they responded strongly to the isolated third harmonics (open bars). In the other cells the two gratings elicited comparable responses.

The above experiment focused on the third harmonics of square waves. However, it was clear from the square-wave tuning of the grating cells that responses to the fifth and higher harmonics were missing as well. In cell $3 \mathrm{BF} 1$ we have also tested gratings composed of thin light or dark lines (rectangular-wave gratings of $8 \%$ and $92 \%$ duty cycle, respectively) and found spatial-frequency tunings very similar in shape to those obtained with square-wave and sinusoidal gratings. In each of these gratings, the fundamental, second, and third harmonics were represented with equal power, but again the cell failed to respond at one-half and one-third the optimum frequency.

The absence of responses to the harmonics of nonsinusoidal gratings is an important finding regarding the function of these

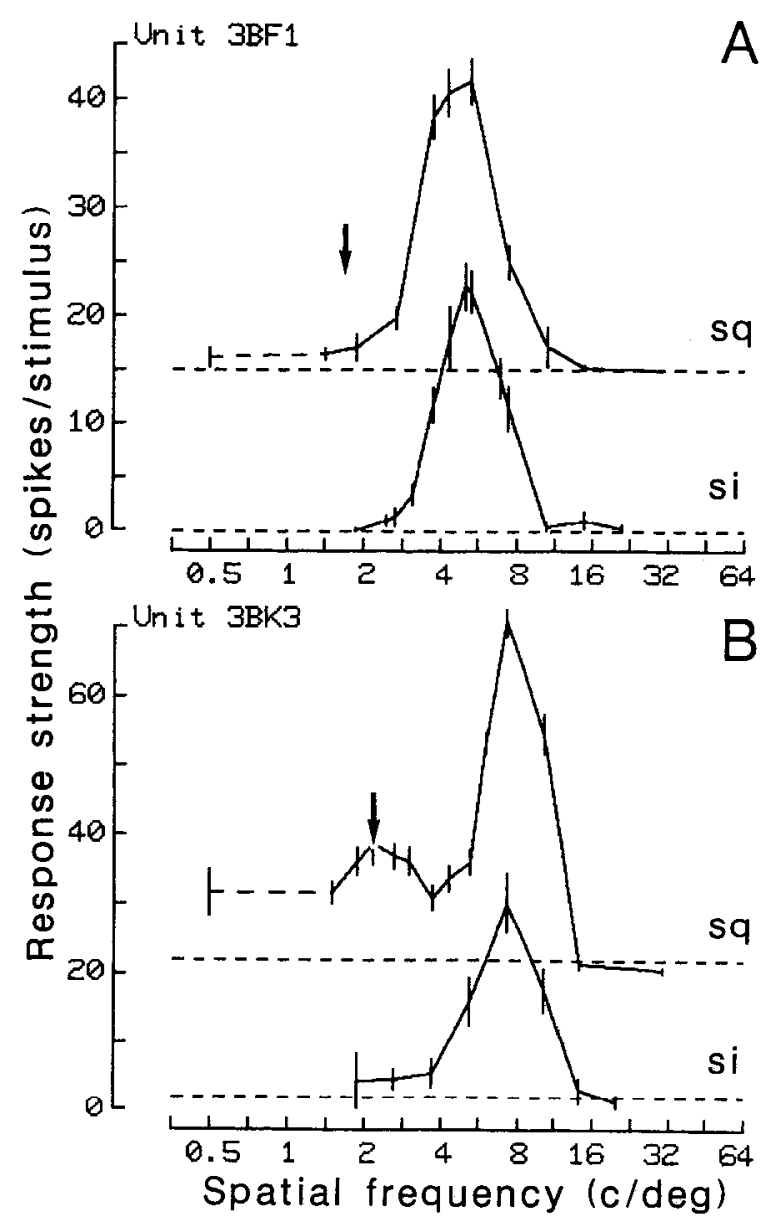

Figure 10. Spatial frequency-response curves obtained with squarewave gratings $(s q)$ and sine-wave gratings $(s i)$ in a grating cell $(A)$ and a "normal" complex cell $(B)$ with similar sine-wave tuning. The squarewave tuning curves have been shifted upward for clarity; broken lines indicate spontaneous activity. Error bars indicate \pm 1 SEM. A secondary peak in the square-wave tuning corresponding to the third harmonic was observed in the "normal" cell, but not in the grating cell (arrows). Cell $A$ : layers 2 and 3 of $\mathrm{V} 1$; bar-grating index, -0.86 ; cell $B$ : layer $4 \mathrm{~B}$ of $\mathrm{VI}$; index, -0.29 . From von der Heydt (1987), with permission.

cells. It confirms our conclusion, drawn from the unresponsiveness to bars and edges, that grating cells do not signal spatialfrequency components. The experiment of Figure 11 has shown that the failure to signal harmonics is not due to lack of sensitivity, but to violation of the superposition principle: the cells responded to a sine-wave grating of the preferred frequency, but when the other harmonic components of the square wave were added, each of which was ineffective by itself, the cells did not respond.

\section{Other textures}

We have shown that grating cells are selective for the periodicity of gratings but do not respond to Fourier components of corresponding spatial frequency in general. This means that these cells must have a rather specialized function. While Fourier components in the "pass-band" of a grating cell occur frequently under normal visual conditions, gratings of the proper periodicity and orientation are rare. This specialization was quite obvious to us when we recorded from the cells, since they rarely fired a single spike during the intervals between fixation periods, 
A

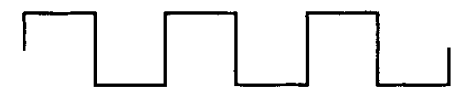

mm

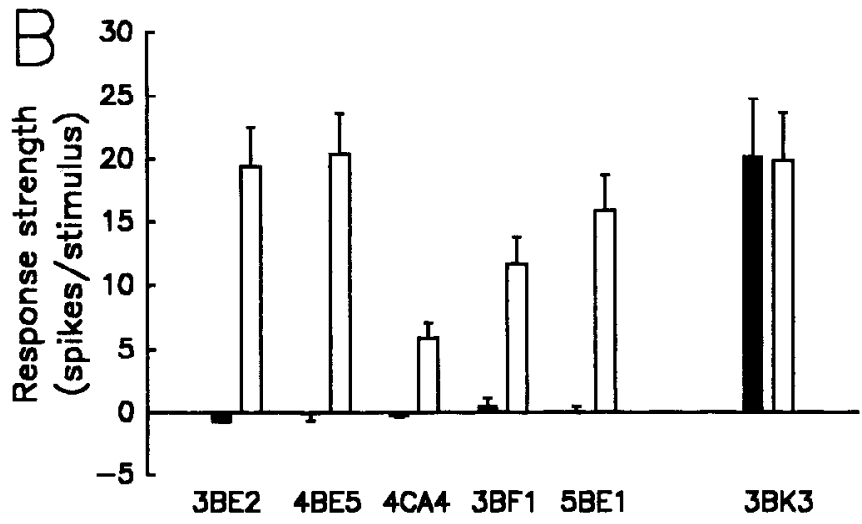

Figure 11. Grating cells do not signal Fourier components. The figure shows a comparison of the responses to a square-wave grating of onethird the preferred frequency with the responses to its isolated third harmonic. $A$, Profiles of the stimuli $B$, Responses of five grating cells ( $3 B E 2$ to $5 B E I$ ) and a "normal" complex cell ( $3 B K 3)$ for comparison. Solid bars, square waves; open bars, third harmonic sine waves. Error bars indicate \pm 1 SEM. The complex cell $3 B K 3$ responded equally to both gratings. In the grating cells, the coarse square-wave gratings produced no responses, although their third harmonics alone were quite effective. Bar-grating index, from left to right $;-1.00,-0.97,-0.93$, $-0.86,-0.84$, and -0.29 .

when the monkey was looking around. We wondered what other patterns, besides gratings might excite these cells.

Figure 12 shows the responses of a grating cell to gratings and various checkerboard patterns. The stimuli are illustrated for some of the data points, as marked by arrows. The cell was tuned to $4.2 \mathrm{c} / \mathrm{deg}$ with a bandwidth of 1 octave (Fig. 12A). Bars and edges had negative effects (mean, 0.3 spikes/sec; mean spontaneous, 1.2 spikes $/ \mathrm{sec}$ ). Figure $12 B$ shows the responses to checkerboard patterns of $4.2 \mathrm{c} / \mathrm{deg}$ horizontal periodicity and various aspect ratios. The cell responded as long as the checks were vertically elongated, but the responses declined with decreasing check length, reaching half-maximal strength with check sizes of $7 \times 30 \mathrm{~min}$ arc $(1 \mathrm{c} / \mathrm{deg}$, third pattern from left $)$ and zero when the checks were approximately square shaped $(4 \mathrm{c} / \mathrm{deg})$. With gratings, the cell showed length summation up to $80 \mathrm{~min}$ arc (and no end-inhibition). It is interesting that the cell responded even when its receptive field was covered by several rows of the pattern, that is, strips of grating with alternating contrast polarities. In fact, all the checkerboard stimuli had odd vertical symmetry relative to the center of the response field so that cqual portions of pattern with opposite contrasts were sampled.

We have tested the effect of vertical positioning with patterns of one and two vertical cycles (the second and third patterns of Fig. 12B). The position was varied in steps of $5 \mathrm{~min}$ arc in pseudorandom order. The result in the first case is shown in Figure $12 C$. There was no "null position"; roughly equal responses were obtained over the entire range of $80 \mathrm{~min}$ arc. A similar result was obtained with the next-finer checkerboard pattern. Thus, one could not locate the lines of phase reversal from the responses of this cell. Grating cells seem to sum sub- units with receptive fields distributed in the length dimension, similar to that found above for the width dimension.

A checkerboard pattern is periodic in various directions, along the rows and columns, along the diagonals, and so on. The diagonal periodicity is particularly obvious when the checks are small. The pattern then looks like two crossed gratings. These gratings correspond in fact to the fundamental Fourier components of the pattern (Kelly, 1976; De Valois et al., 1979). We have tested the cell of Figure 12 with checkerboards of various frequencies and orientations. When the checks were nearly square and the periodicity of the rows matched the cell's preferred frequency $(4 \mathrm{c} / \mathrm{deg}$ ), the cell failed to respond (cf. Fig. 12B). When the pattern was expanded so that the spacing of the diagonals matched its preferred frequency, the cell responded. For this checkerboard, the orientation tuning showed two maxima $80^{\circ}$ apart, corresponding to the orientations of the diagonals (Fig. 12D). Thus, the cell again seemed to signal the fundamental components, as was found in the one-dimensional case for gratings.

Orientation versus direction of periodicity. The periodicity of a grating is usually defined along the axis orthogonal to its orientation. Therefore, a cell's orientation tuning measured with gratings may reflect selectivity either for orientation or for periodicity. In two-dimensional patterns, the axes of periodicity are in general not orthogonal to the orientations of the fundamental components, and we can thus distinguish whether the tuning is for orientation or periodicity. The left and right stimulus insets of Figure 12D show the stimuli at the orientations of maximum responses, defined as the midpoints of the peaks at half-height (left and right arrows). One can see that in either case one set of diagonals is vertical, while the effective periodicities are not exactly horizontal. Thus, the orientation seemed to be more critical than the direction of periodicity. With patterns of elongated checks, the cell responded when the rows had the preferred horizontal periodicity (Fig. 12B), but for these patterns the diagonals were also close to the vertical and their spacing corresponded nearly to the preferred periodicity, so that the responses could also be attributed to the fundamental components.

In conclusion, we have learned from the experiment with checkerboards that (1) grating cells may respond to periodic patterns other than gratings, even when there are contrast reversals in the length direction within the receptive field; and (2) they seem to signal frequency and orientation of the fundamental components of two-dimensional periodic patterns as well as gratings, and may do this even in the presence of another strong component of different orientation.

Stresemann patterns. In order to see how critical the requirement of the proper periodicity is, we have tested a grating cell with patterns derived from a grating of optimum periodicity by displacing every other light bar to one side. A grating in which the light bars were thinner than the dark bars ( $35 \%$ duty cycle) was used. Examples are shown, with reversed contrast, in the stimulus insets of Figure 13. We call these "Stresemann patterns" after the famous trousers of the famous German statesman. The distance between adjacent bars alternates between values smaller and greater than the optimum. The responses of the grating cell are plotted in Figure 13 as a function of the displacement, expressed in fractions of the preferred grating cycle ( $20 \mathrm{~min}$ arc). The responses declined gradually, approaching zero somewhere near 0.4 . The cell tolerated displacements up to about 0.14 , which corresponds to a $3: 4$ jitter in bar spacing. 

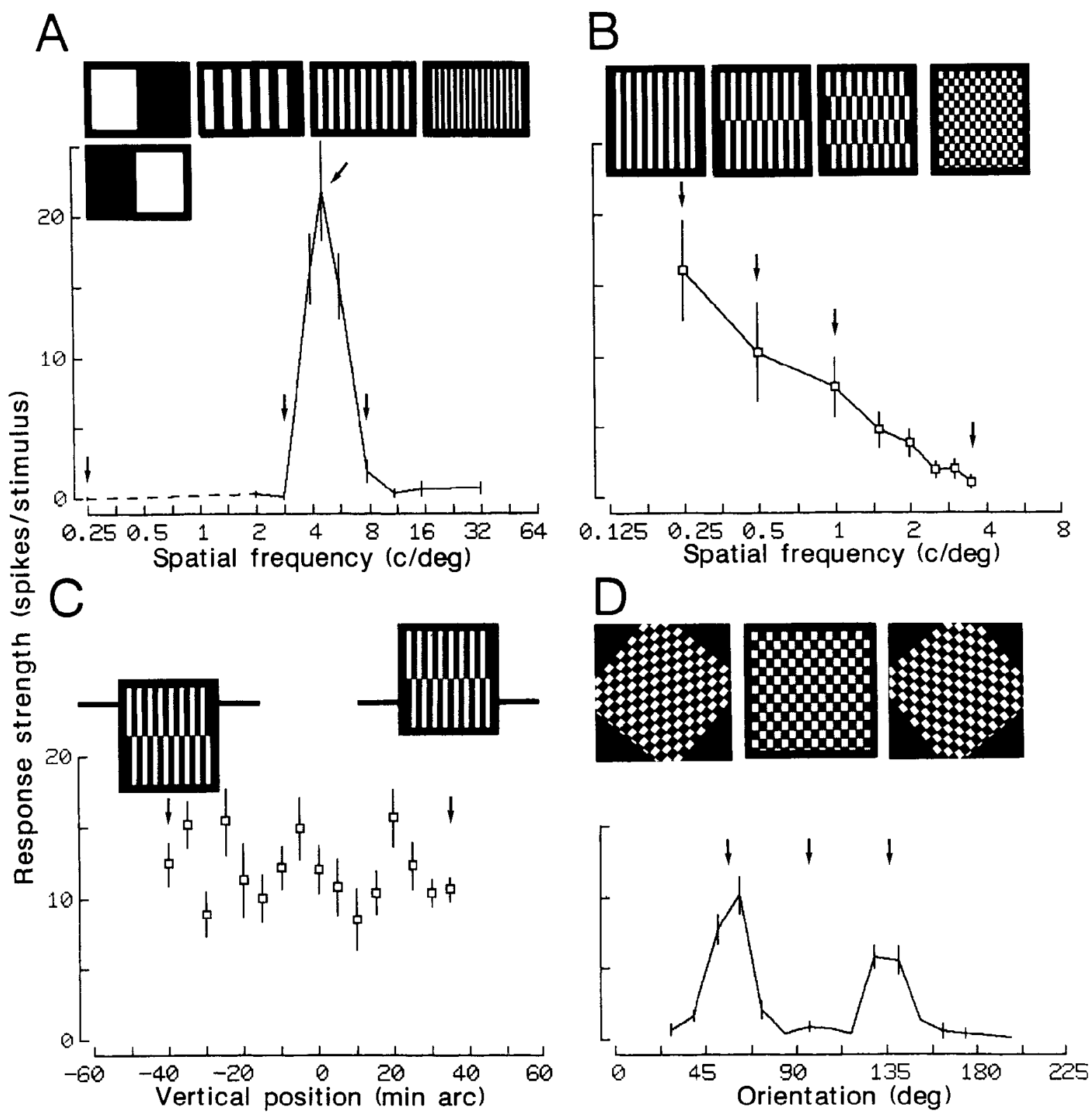

Figure 12. Responses of a grating cell to square-wave gratings and checkerboard patterns. Stimulus insets refer to data points marked by arrows. $A$, Spatial-frequency tuning obtained with square-wave gratings. The peak frequency was $4.2 \mathrm{c} / \mathrm{deg}$; the "bandwidth," 1.0 octaves. $B$, Responses to checkerboard patterns of the preferred periodicity in horizontal direction and varied vertical check sizes. Spatial frequency on the abscissa here refers to the vertical periodicity. The cell responded as long as the checks were elongated. $C$, The effect of shifting the line of contrast reversal. There was no indication of a null position. $D$, Orientation tuning with a checkerboard pattern of check periodicity 1.41 times the preferred grating periodicity. Peaks of response were found at the orientations of the diagonals. Error bars indicate \pm 1 SEM. All stimuli are shown to scale, those of $A$ with half their original horizontal extent. For ease of comparison, all patterns have been rotated $6^{\circ}$ clockwise, as if the receptive field orientation had been vertical. The cell was recorded in layers 2 and 3 of V1; field at $1.8^{\circ}$ eccentricity; bar-grating index, -1.03 .

The cell was also sensitive to the duty cycle of rectangularwave gratings of optimum periodicity. The response was maximum for $55 \%$ duty cycle and reached zero at $10 \%$ and $85 \%$ (gratings of thin light or dark bars, respectively). This is interesting because the same cell responded invariantly over a wide range of contrasts (Fig. 14, cell 5BE1). Since reduction of contrast and reduction of duty cycle both reduce the modulation of the fundamental component, linear filter theory would predict that the responses declined similarly, but this was obviously not the case. For instance, a grating of $85 \%$ duty cycle and $40 \%$ contrast produced almost no response $(1.3 \pm 0.6$ spikes $/$ stimulus). The fundamental of this grating had a modulation of $23 \%$, but square-wave gratings with the same modulation of fundamental evoked strong responses $(17.3 \pm 1.6$ spikes/stimulus).

\section{Contrast sensitivity}

In studies of human perception using gratings it is often assumed that the visual system is linear at low contrast, so that, for example, detection of one spatial-frequency component would not depend on the presence of other components as long as the 


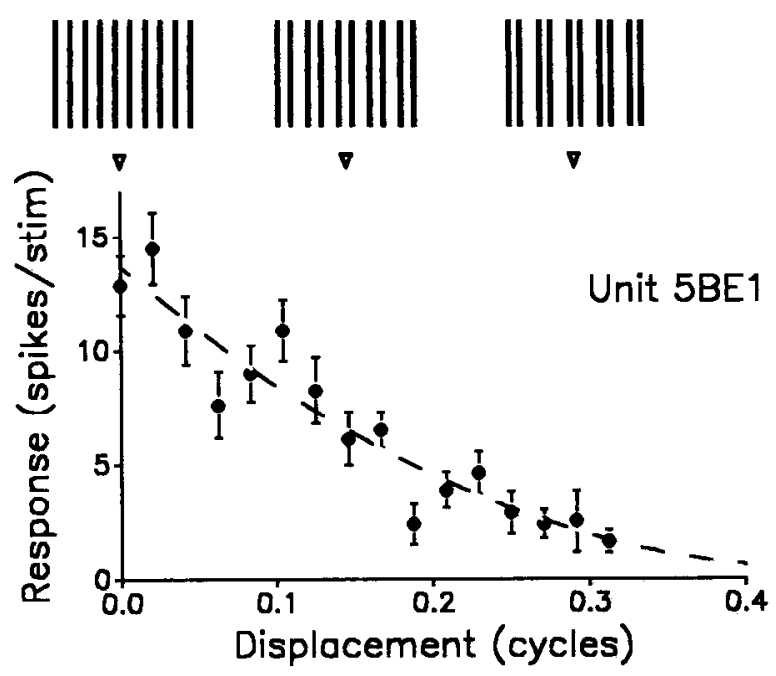

Figure 13. Responses of a grating cell to "Stresemann patterns." In a grating of the preferred periodicity, every other bar was displaced by a fraction of a cycle, as indicated on the abscissa. The responses decreased gradually with increasing displacement. The broken line is a quadratic regression line. Grating width, $4^{\circ}$; length, $0.5^{\circ}$. The cell was recorded in layers 2 and 3 of V1. Spontaneous activity, 0.16 spikes/stimulus; endstopped field at $2.6^{\circ}$ eccentricity; preferred periodicity, $3 \mathrm{c} / \mathrm{deg}$; width of tuning, 1.3 octaves; bar-grating index, -0.84 .

contrast of cach component is not much higher than its threshold value, which has in fact been demonstrated (e.g., Campbell and Robson, 1968). The conclusion must be that nonlinear elements like grating cells, if they exist also in the human visual system, were not involved in the measurements of those studies. Either they are silent at low contrast or they become linear.

Because of their selectivity and nonlinear filter properties, we expected that grating cells would be insensitive at low contrast. Indeed, summation-to-threshold mechanisms (McCulloch and Pitts, 1943) were often assumed to explain selectivity and nonlinearity of cortical neurons (cf. Schumer and Movshon, 1984; De Valois et al., 1985). The threshold nonlinearity would make such neurons unresponsive at low contrast. The responses to gratings with varied number of bars (Fig. 6) also suggested a threshold nonlinearity. However, surprisingly, the grating cells showed no pronounced contrast thresholds. On the contrary, some of them responded even to gratings of very low contrast.

In Figure 14 we have plotted the contrast-response functions of four grating cells on a logarithmic scale. It can be seen that some cells reached saturation already at a few percent contrast; others, only at $50 \%$. Thus, it seems that some grating cells are dominated by magno- and some by parvocellular input (Shapley et al., 1981). The most sensitive cell (SBE1) was virtually "turned on" at a very low contrast, little more than $1 \%$, and saturated at only $3 \%$. Over a wide range of contrast, from $3 \%$ upwards, its responses remained nearly invariant. This remarkable switching characteristic underlines the proposed function of these cells, namely, to detect periodic patterns and signal periodicity, a task that should not depend on contrast.

Analysis of its receiver operating characteristic (Green and Swets, 1966; see also Tolhurst et al., 1983) showed that this cell discriminated a contrast of $1.7 \%$ from any of the lower contrasts $(0.4-1.2 \%)$ correctly in $93 \%$ of the presentations. (Contrasts between $0.4 \%$ and $42 \%$ were tested in series in random order, while the spontaneous activity was determined separately; comparing $1.7 \%$ with zero contrast would have exaggerated the re-

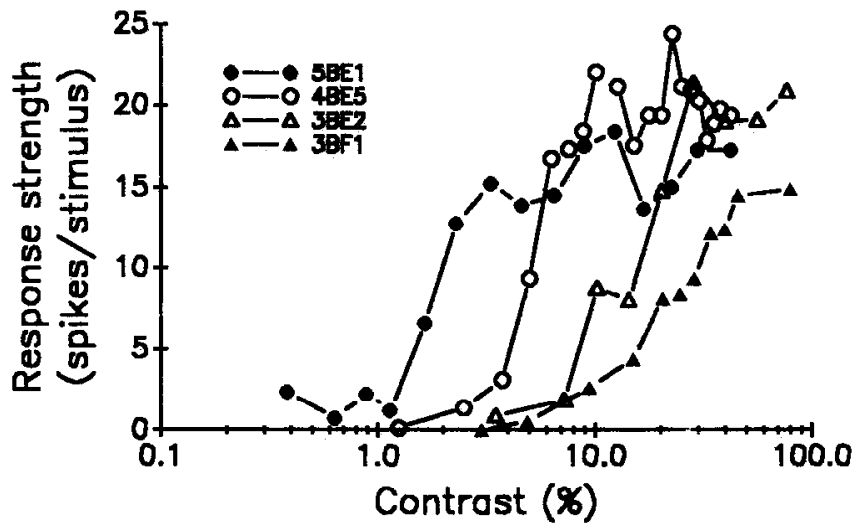

Figure 14. Contrast-response functions of four grating cells. The mean numbers of spikes per stimulus cycle are plotted as a function of the modulation $\left(L_{\max }-L_{\min } / L_{\max }+L_{\min }\right)$ of the fundamental component of the gratings on logarithmic scale. Sinusoidal gratings were used for cells $4 B E 5,3 B E 2$, and $3 B F 1$; square-wave gratings for $5 B E 1$. Periodicities were $10,5,4.4$, and $3 \mathrm{c} / \mathrm{deg}$; amplitudes of movement, $48,32,35$, and $48 \mathrm{~min}$ arc; and frequencies of movement, $1,1,1.5$, and $1 \mathrm{~Hz}$, respectively. The cells had bar-grating indices of $-0.97,-1.00,-0.86$, and -0.84 .

sult.) This means that the cell was about as sensitive as the animal, since the $75 \%$ correct threshold of macaque observers is slightly above $1 \%$ at $3 \mathrm{c} / \mathrm{dcg}$ (De Valois et al., 1974). Note that our procedure was not optimized for demonstrating maximum sensitivity because we presented widely different contrasts in random sequence. Experiments in the cat have shown that cortical cells become more sensitive after a period of stimulation with only low contrast ("contrast gain control"; Ohzawa et al., 1982; Albrecht et al., 1984). In conclusion, if humans have grating cells, they can probably use them even at contrasts near threshold.

We have considered also the other possibility, that the nonlinearity of grating cells vanishes at low contrast. In Figure 15 we have plotted the amplitudes of the responses of grating cell $3 \mathrm{BF} 1$ (preferred frequency, $5 \mathrm{c} / \mathrm{deg}$; bandwidth, 1.2 octaves) to various line gratings. The lines were $1 \mathrm{~min}$ arc thick, and their spacing was varied. Figure 15 shows the responses to gratings of $1,1 / 2$, and $1 / 3$ the preferred frequency at two contrast levels. To the right are shown the amplitude spectra of the three gratings. Either the first, the second, or the third harmonic coincided with the cell's preferred frequency (asterisks). Since the bars were thin, the successive harmonics of each grating had nearly constant amplitudes. However, because line width was kept constant, the lower-frequency gratings had smaller amplitudes due to their lower duty cycles. The responses corresponding to first, second, and third harmonics are represented by circles, diamonds, and triangles, respectively. The spontaneous activity is also represented by a circle. One can see that the circles fall on a straight line; that is, the responses increased linearly with the amplitude of the fundamental. However, it is clear also that the triangles and diamonds fall below this line. This means that the higher harmonics are not represented by responses corresponding to their amplitudes, even at these low levels of modulation. Thus, grating cells do not become linear at low contrast.

Neuronal responses are often said to be ambiguous. For example, a non-optimally oriented stimulus at high contrast may give the same response as an optimally oriented stimulus of low contrast. In the case of grating cells, one may ask whether a few 


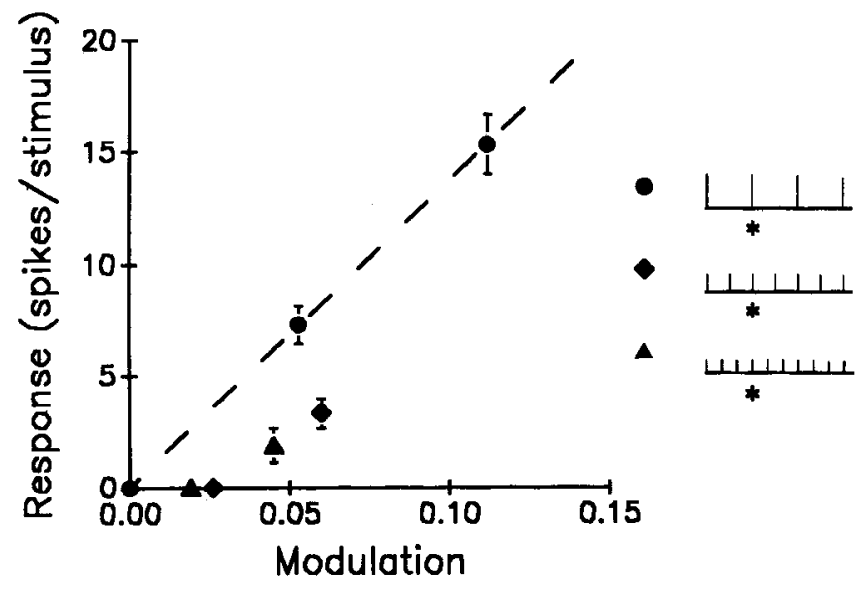

Figure 15. Nonlinearity at low contrast. The responses of a grating cell at the first, second, and third harmonic frequencies of gratings of thin lines. The Fourier spectra are shown to the right; asterisks indicate the preferred frequency. Each of the gratings was presented at two contrasts. The responses are plotted versus the modulation of the respective Fourier components. Circles, first harmonic; diamonds, second harmonic; triangles, third harmonic; circle at zero modulation, no pattern. The cell signaled the first, but not the higher harmonics. The cell was recorded in layers 2 and 3 of V1; bar-grating index, -0.86 ; preferred frequency, $5 . \mathrm{c} / \mathrm{deg}$ : "bandwidth," 1.2 octaves. The gratings had $1 \mathrm{~min}$ arc line width and periodicities of 12,24 , and $36 \mathrm{~min}$ arc.

bars of high contrast may give the same response as a full grating of low contrast. Two or three bars are not perceived as a texture, but a grating appears as a periodic texture independently of contrast. In Figure 16 we have plotted, in the same graph, the results of varying numbers of bars and contrast in four grating cells. Open circles and solid lines show the responses to fixed contrast gratings of various extents, as indicated on the abscissa; solid circles and broken lines show the responses to gratings of fixed extent as a function of contrast. The contrast curves are replotted from Figure 14, except that the contrast values have been scaled for each cell so that the last point of the curve was plotted at the number of cycles used. Thus, each pair of curves shows the result of reducing either the contrast or the number of cycles of a particular grating. One can see that this had different effects. When the number of cycles was reduced the responscs gradually approached zcro, but when the contrast was reduced, they remained constant over a fairly large range and finally dropped. We conclude that grating cells signal the presence of a periodic pattern to some extent unambiguously. With the proper grating, the responses are largely independent of the contrast, but when the number of cycles is reduced below a critical value, the responses drop.

\section{Discussion}

We have described a type of neuron in monkey visual cortex that responded to gratings and other periodic patterns, but not to single bars or edges. The distribution of a bar-grating response index indicated a separate group of cortical neurons in which light and dark bars produced nearly zero responses or had even

Figure 16. Comparison of the effects of varying the contrast and varying the number of cycles of a grating in the four grating cells of Figure 14. Open circles, responses to square-wave gratings of fixed contrast and varied number of cycles; solid circles, contrast-response functions with
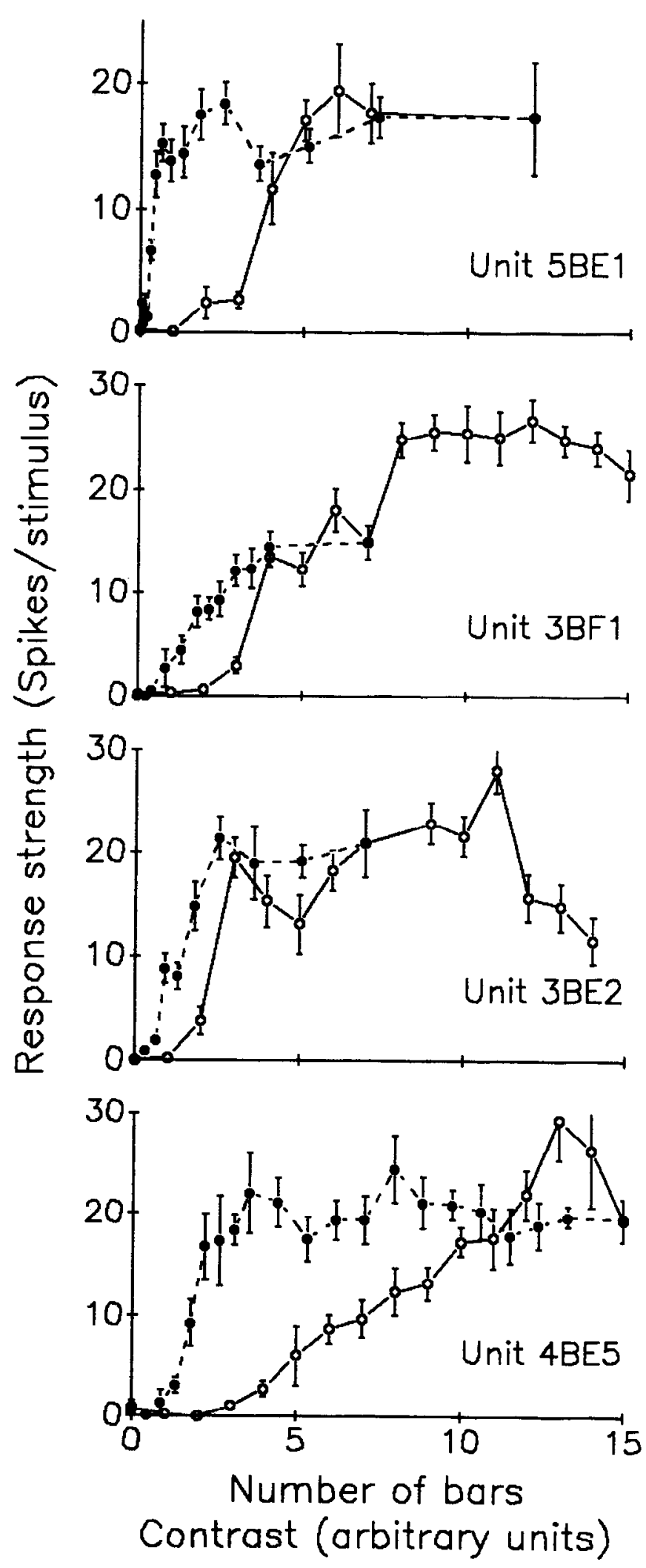

fixed number of cycles. The common stimulus of the two series was used to scale the abscissae of the contrast series. When the extent of the grating was reduced below a critical number of cycles, the responses dropped, but when the contrast was reduced, responses to a grating of given extent remained invariant over some range. Periodicities, from top to bottom: $3,4.4,5$, and $10 \mathrm{c} / \mathrm{deg}$; contrast (if varying number of cycles); $42 \%, 80 \%, 77 \%$, and $42 \%$; number of cycles (if varying contrast): $12,7,7$, and 15 , respectively. 
inhibitory effects. We found that these cells were more sharply tuned (median of 1 octave for V1) and preferred higher spatial frequencies (median of $9 \mathrm{c} / \mathrm{deg}$ for $\mathrm{Vl}$ ) than other cells on average. They showed extensive summation in terms of grating cycles, with a median of 7.5 , which is unusual (cf. De Valois et al., 1985). They responded to moving as well as stationary gratings, usually independently of the grating phase, and nearly always without preference for the direction of motion. Grating cells were more frequent in $\mathrm{V} 1(4 \%)$ than $\mathrm{V} 2(1.7 \%)$. In $\mathrm{V} 1$, they were concentrated in the supragranular layers, and remarkably, many of them were monocular.

The finding that most surprised us, however, was that these cells did not signal the spatial Fourier components of the stimulus, but seemed to be specialized for the detection of patterns that are really periodic in space: they failed to respond to bars and edges (Figs. 1, 2), although from the Fourier spectra of these stimuli (Fig. 9) and the ranges of grating periodicities over which the cells responded, one would have expected significant responses. With square-wave and line gratings, the cells signaled the fundamental component, but not the higher harmonic components (Figs. 10, 11, 15).

When cells do not respond where they are expected to respond, one can think of several possible explanations. We need not consider effects of anesthesia since no drugs were used in these experiments. Also, the condition of the cortex is a minor concern since our animals were performing a visual task that involved a part of the visual cortcx that was close or cyen overlapping with the region that was studied, and all did very well. The responses might be influenced by factors like attention, and so on. One could think, for example, that the fixation task itself caused a reduction of responses to parafoveal or even foveal stimuli that are irrelevant to the task. However, this can hardly be the explanation for the failure to signal certain Fourier components because sinusoidal gratings exactly equal to those harmonics, presented alone, elicited strong responses (Fig. 11). Also, the contrast sensitivity of some grating cells (Fig. 14) was as high as the sensitivity of the animal known from behavioral studies.

The results obtained with gratings of different profiles as well as the bar-grating comparison demonstrate that the responses of grating cells do not conform to the superposition principle, the definition of linear systems. For a linear spatial filter, it makes no difference whether a sinusoidal grating of frequency $3 f$ is presented alone or superimposed on other gratings of frequencies $f, 5 f, 7 f$, and so on, making up a square-wave grating, if only the frequencies of the additional gratings are outside its frequency pass-band. This is true not only for linear filters, but also for devices with linear spatial summation and an output nonlinearity (e.g., rectification, or a threshold), and even for systems that sum the output of several such devices, which is commonly assumed to be the mechanism of complex cells ( $\mathrm{Hu}-$ bel and Wiesel, 1962; Movshon et al., 1978b; De Valois et al., 1985). Since the results of the initial spatial summation are identical for the sinusoid and the compound grating, so must be the outputs. This has in fact been demonstrated in some cortical cells. Maffei et al. (1979) and Pollen and Ronner (1982) have shown examples of simple and complex cells from the cat's striate cortex in which a square-wave grating of one-third the optimum frequency and its isolated third harmonic produced equal responses. We have shown the example of a complex cell of V1 (cell 3BK3, Figs. 10, 11). The grating cells behaved differently.
High-frequency components as contained in the Fourier spectra of square-wave gratings, bars, and edges are important for perception and therefore have to be taken into account by the machinery of $\mathrm{V} 1$, which in monkey and man is the gate for visual information to the cortex. The conclusion drawn here is that the cells we call grating cells, although selective for spatial periodicity, are not doing anything that resembles spatial-frequency analysis and are therefore functionally different from the cortical cells showing spatial filter properties.

\section{The definition of grating cells}

The comparison between bar and grating responses and the definition of neuronal "preferences" is somewhat arbitrary (cf. Albrecht et al., 1980; Stork et al., 1982). First, the equalization of contrast is a matter of definition. We have chosen bar and grating profiles of equal peak-to-peak luminance modulation (the surround of the bars equalled either the minimum or the maximum luminance of the gratings). Other studies have used half the modulation for the bars, matching the bar surround to the mean luminance of the gratings (Albrecht et al., 1980). Our choice should favor the bars, if anything. Second, since the stimulus energy is more localized in bars than in gratings, how much of it is sampled by a unit depends on the size of its receptive field and the stimulus movement. We have oscillated both stimuli back and forth with small amplitude (usually equal to two cycles of the grating), sweeping the bars over the most sensitive part of the receptive field. The exact definition of preference is probably not very important in the present study since the responsiveness to single bars of grating cells was so much lower than that of the other cells. One could perhaps use other criteria to define grating cells, but our criterion is simple and does not require sophisticated stimulation. It seems to define a rather homogeneous population of cortical cells.

\section{Grating cells have not been described previously}

Bars and gratings have been used in many previous studies of the visual cortex, but grating cells have not been described. In the striate cortex of anesthetized, paralyzed cats, Movshon et al. (1978b) "... noticed no overall difference in the vigor with which cells responded to moving gratings as opposed to bars or edges" and do not mention cells unresponsive to bars and edges. Schiller et al. (1976) found that many cells in anesthetized monkey striate cortex "responded poorly to sine-wave gratings cven when bars and edges elicited good responses from them" and do not mention the opposite. Albrecht et al. (1980) specifically compared the strengths of bar and grating responses in anesthetized cats and monkeys. For the ratio of grating to bar responses, they found means and standard deviations of $2.2 \pm$ 0.7 for simple cells and $1.8 \pm 0.8$ for complex cells. Thus, both types responded about two times better to gratings than to bars. However, the typical grating cell of Vl had a bar-grating response index of -0.95 (cf. Fig. 2), which corresponds to a ratio of grating to bar responses of 39 . From the quoted means and standard deviations, using Chebyshev's inequality, which holds independently of the type of distribution, this ratio has a probability of less than one in two thousand among either the simple or the complex cell group. This shows that there was no grating cell in the sample of Albrecht et al. (1980) and indicates how far these cells differ from the "normal" population. [Thcre are differences in method of stimulation and evaluation of responses. However, we think these differences have only minor influence since for the control sample of $\mathrm{Vl}$ we obtained a 
median index of -0.22 , corresponding to a grating-to-bar response ratio of 1.6, which is close to the values of Albrecht et al. (1980).] Poggio et al. (1977) have studied the striate cortex in the alert, behaving monkey using square-wave gratings. Interestingly, their Figure 11 shows one spatial-frequency tuning curve with the simple, unimodal shape that we found in the grating cells, while the other three curves show the additional peak corresponding to the third harmonic of the gratings (cf. our Fig. 10). Thus, it seems possible that grating cells have not been reported earlier because the other studies have used the conventional, anesthetized preparation. Since the layers 2, 3, and $4 \mathrm{~B}$ of $\mathrm{V} 1$ send information to other cortical areas, summarizing the processing of $\mathrm{V} 1$, cells in these layers are prohahly most susceptible to the effects of anesthesia. The superficial localization of most grating cells also makes them vulnerable to alterations of their environment that might be caused by removal of the dura, which is the rule in anesthetized preparations. Conceivably, a degradation of inhibitory connections makes the grating cells lose their selectivity and appear similar to common complex cells.

Exclusive bar responses. We have seen also cells that responded to bars but not to gratings. These are represented near +1 on the abscissa in Figure 2. The responses of such a cell to gratings of varied number of bars was shown in Figure 6. These cells responded selectively to narrow bars, either light or dark, and were not excited by edges. We do not know how frequent they are because our control sample is limited and most of the other cells were not tested with gratings of all frequencies. Schiller et al. (1976) found cells in V1 that responded to bars or edges but could not be activated by sinusoidal gratings. Unresponsiveness to square-wave gratings of any periodicity is even more surprising. It indicates that the reason why cells fail to respond to sinusoidal gratings is not the lack of sharp contours, but the repetitive character of the pattern.

\section{Spatial-frequency tuning}

Previous studies of the monkey striate cortex indicated that the distribution of preferred spatial frequencies of foveal and parafoveal neurons has a maximum between 3 and $5 \mathrm{c} / \mathrm{deg}$ and falls off steeply at high frequencies (Poggio et al., 1977; De Valois et al., 1982; Foster et al., 1985). Our control sample of VI showed a similar distribution (median of $6 \mathrm{c} / \mathrm{deg}$ ), while that of the grating cells was centered at higher frequencics (mcdian of 9.3 $\mathrm{c} / \mathrm{deg}$ ). The proportion of grating cells is therefore higher among cells with high preferred frequencies. From De Valois et al. (1982), their Figure 7, we calculate that less than $10 \%$ had peak frequencies greater than $8 \mathrm{c} / \mathrm{deg}$, compared to more than $50 \%$ of the grating cells.

In normal as well as grating cells, we found that over the range of $4^{\circ}$ the preferred frequencies did not vary much with retinal eccentricity, compared to the scatter at each eccentricity, as was found by De Valois et al. (1982).

A bandwidth at half-amplitude of 0.43 octaves, as measured for cell 2DN2, is perhaps the smallest found so far in the monkey, and the median bandwidth of grating cells of 1 octave is considerably smaller than the 1.4 and 1.5 octaves found by De Valois et al. (1982) for simple and complex cells, respectively, and the 1.7 octaves found by Foster et al. (1985) in V1. On the other hand, these values agree with the median bandwidth of 1.5 octaves of our control sample. De Valois et al. (1982) determined the width of contrast sensitivity functions for fixed criterion responses, whereas Foster et al.'s (1985) measure- ments, as ours, were based on response functions obtained with fixed contrast. While the cited studies have used sinusoidal gratings, we have used square-wave gratings. This should have increased our estimates of the bandwidths (cf. Schiller et al., 1976). On the other hand, the exclusion of cells that did not show a low-frequency cutoff, which could be due to the use of squarewave gratings (cf. Schiller et al., 1976; Poggio et al., 1977), may have biased our comparison sample toward more narrowly tuned cells.

In conclusion, our results in "normal cells" of V1 agree with the previous studies, while the "grating cells" are more narrowly tuned and prefer higher frequencies than the previously reported cells.

\section{The role of grating cells in vision}

The failure to conform to the superposition principle means that one cannot predict the responses to complex stimuli from a set of responses to simple stimuli. The ided of plotting receptive fields is that the map obtained from the responses to spots of light, bars, or edges has a meaning for the function of a cell under the more complex natural conditions of stimulation. This is not so in grating cells, where the reaction to a single bar was in no way related to the responses obtained with a few parallel bars. Similarly, the spatial-frequency tuning of the cells obviously does not enable us to predict their responses to bars and edges. A quantitative nonlinear model has to be worked out. Inhibitory interaction between spatial-frequency channcls has been invoked to explain deviations from linearity found in cells of the cat striate cortex (De Valois and Tootell, 1983). Whether this explanation can also account for the behavior of grating cells will have to be shown by simulation, alternative models will have to be discussed, and more experiments are probably needed to decide the issue. In any case, whatever the exact mechanism may be, it is clear that spatial summation in grating cells is highly nonlinear and thus cannot be described even approximately as linear filtering. The question of how the nonlinearity is actually implemented is irrelevant for the interpretation of the function of the cells.

We suggest that grating cells signal patterns that are perceived as periodic. It seems to us that humans have a distinct perception of visual periodicity, and we conjecture that this perception is related to the activity of the kind of cell we have demonstrated in the monkey.

At first glance, the results obtained with different profiles of gratings and with checkerboard patterns seem to be consistent with the assumption that grating cells signal the fundamental frequency components of periodic patterns. When the responses were plotted against the fundamental frequencies, the tuning curves for sinusoidal and square-wave gratings were similar (Fig. 10). A checkerboard pattern produced the best responses when its fundamentals matched that of the optimum grating in frequency and orientation (Fig. 12). When the fundamental was attenuated by varying the duty cycle of rectangular-wave gratings, or by adding jitter to the bar positions, the responses were reduced (Fig. 13). However, contrary to this assumption, reduction of contrast, which also attenuates the fundamental, did not affect the responses (Fig. 14, cell 5BE1). As a consequence, rectangular-wave gratings and square-wave gratings of the same amplitude of modulation of the fundamental evoked quite different responses, almost zero and maximum. This result shows again that the nonlinearity of grating cells is not just a matter of a nonlinear amplitude-response function. In conclusion, one 
cannot predict the responses of grating cells to periodic patterns from the amplitude of the fundamental component.

Another issue to be discussed is that area V1 is usually conceived as performing a fairly general, preliminary, processing of visual information. Spatial frequency filtering is such a general task. Since cortical cells are not too narrowly tuned for spatial frequency and orientation, and since most natural stimuli have distributed Fourier spectra, a cell of the linear filter type will be active almost continuously, to a varying degree, under normal conditions. However, few stimuli are periodic, and patterns of the periodicity and orientation required by a grating cell will appear rarely. Thus, we are forced to assume a rather specialized function for a sizable number of cells in primary visual cortex. On the other hand, for most grating cells the selectivity in the domains of spatial frequency and orientation was not extreme. A bandwidth of one octave at half-amplitude means that a cell responds to gratings that differ in periodicity up to a factor of about 2.8 . Therefore, it would probably respond also to slightly irregular striped patterns. The results with "Stresemann patterns" (Fig. 13) support this assumption. Furthermore, grating cells seem to respond not only to one-dimensional patterns like gratings, but also to other periodic textures like checkerboard patterns (Fig. 12). Thus, it seems likely that each of these cells can be excited by a variety of textures. Finally, the patterns that excite grating cells, although not abundant, may be particularly significant in natural environments. Regular textures often have biological origin (cf. De Valois and De Valois, 1988, p. 337ff). One can easily imagine many objects of importance for primates that are characterized hy a quasi-periodic striped appearance, from a bunch of bananas to snakes and wildcats. Even the fur of a monkey has texture that could possibly drive a grating cell. As a parallel one may think of acoustics, where the sounds (i.e., periodic stimuli) are a small but biologically important subclass of all the acoustic stimuli. Another interesting aspect of periodicity detection is the role of textures in depth perception. Quasi-periodic textures are often due to statistically homogeneous physical structures such as the gravel on a beach or the leaves of a tree. The system can use this knowledge to infer depth and surface slant if it is able to detect differences and gradients in scaling of the visible texture, and it may use the spatial frequency selectivity of grating cells for that purpose.

The conclusion that grating cells signal a quality of texture, rather than Fourier components, is consistent with the observation of unmodulated responses to moving gratings. In general, the responses lacked phase information (which is essential for Fourier analysis). Also, the finding that the median grating cell samples gratings over as many as 7.5 cycles is significant. We have shown that the spatial-frequency tuning of grating cells could be achieved with one-sixth of their receptive field size, on the average, if optimal (Gabor-function) linear filters were used. Grating cells could have a much better spatial resolution. If local spatial frequency analysis were their function, optimal resolution in both frequency and space would be important (cf. Marcelja, 1980). On the other hand, extensive spatial summation makes sense for the analysis of texture, where it can improve the sensitivity or signal-to-noise ratio.

The question remains why a class of cells with a rather specialized function is found in the primary visual area. Our results indicate that grating cells are even more frequent in V1 than in V2. The answer is probably that the analysis of texture requires high spatial resolution, with a correspondingly high load on the processing device. In a point-by-point representation, texture information is highly redundant, calling for a more efficient encoding at an early stage. We have a similar situation in stereopsis, where the demands on spatial resolution are extremely high, and again we found a small proportion of specialized cells in V1, cells responding exclusively to binocular stimulation with stereoscopic disparity (Poggio, 1984). In this context, it is significant that grating cells in V1 were often driven from one eye only and were nearly always insensitive to direction of motion, indicating again that periodicity is encoded early, independently of, and in parallel with other stimulus features such as direction of motion and binocular disparity.

\section{Implications for psychophysics}

If one can generalize results obtained in the monkey to human vision, it is likely that judgments of grain and orientation of certain textures, and possibly also their detection, involve the responses of grating cells. By the signals of such cells one could distinguish periodic or quasi-periodic patterns from aperiodic patterns, whereas cells of the linear filter type give more ambiguous signals since they respond also to lines, sharp edges, and so on. Grating cells do not participate in a general transformation of the image, but rather work on a particular aspect, in parallel with other channels.

It is possible that some of the psychophysical experiments designed to reveal and characterize the spatial-frequency channels of Campbell and Robson (1968) in fact measured the performance of grating cells. We cannot revicw here the cxtensive literature on perception of gratings. An example may illustrate the point. Inspection of a suprathreshold sinusoidal grating raises the contrast threshold for detecting a subsequently presented grating of similar orientation and frequency (Pantle and Sekuler, 1968; Blakemore and Campbell, 1969). If this effect were due to adaptation of spatial-frequency channels, adaptation to gratings of nonsinusoidal luminance profiles should produce effects at the frequencies of the harmonics. However, in forced-choice detection experiments, no threshold elevation at the third harmonic was found after adaptation to square-wave gratings (Nachmias et al., 1973), a result that could not be explained. Since grating cells do not respond to the third harmonics of square-wave gratings (Fig. 11), they are probably not adapted by them either, and if the subjects relied on the signals of such cells, one would expect no adaptation effect. It would be interesting to reexamine the various cxpcriments on perception of gratings under the aspect of whether or not grating cells might have heen involved. Also, one could perhaps design tests that specifically favor or exclude the use of these nonlinear elements. For example, tasks in which subjects have to discriminate gratings from other patterns are likely to favor grating cells, compared to simple pattern detection tasks.

\section{Appendix}

Spatial summation and frequency bandwidth of Gaussian filters

A "Gaussian filter" or "Gabor function" with the center frequency $\Omega_{0}$ and the spread $\sigma$ in the dimension $x$,

$$
g(x)=\exp \left[-x^{2} / 2 \sigma^{2}\right] \cdot \exp \left[i \Omega_{0} x\right]
$$

has the Fourier spectrum

$$
G(\Omega)=\sqrt{2 \pi} \cdot \sigma \cdot \exp \left[-\left(\Omega-\Omega_{0}\right)^{2} \sigma^{2} / 2\right] .
$$

Let $\Omega_{1}$ and $\Omega_{2}$ be the cutoff frequencies at half-amplitude, $G\left(\Omega_{i}\right)$ $=1 / 2 G\left(\Omega_{0}\right), i=1,2$. From Equation $\mathrm{Al}$ we have 


$$
\exp \left[-\left(\Omega_{i}-\Omega_{0}\right)^{2} \sigma^{2} / 2\right]=1 / 2
$$

or

$$
\left(\Omega_{i}-\Omega_{0}\right)^{2}=2 \cdot \ln 2 / \sigma^{2}
$$

which gives

$$
\Omega_{1}=\Omega_{0}+\sqrt{2 \ln 2} / \sigma \text { and } \Omega_{2}=\Omega_{0}-\sqrt{2 \ln 2} / \sigma,
$$

and the bandwidth in octaves

$$
B_{\mathrm{oct}}=\log _{2} \Omega_{1} / \Omega_{2}=\log _{2} \frac{\Omega_{0}+\sqrt{2 \ln 2 / \sigma}}{\Omega_{0}-\sqrt{2 \ln 2 / \sigma}} .
$$

Consider a receptive field of sensitivity profile $A \cdot g(x)$ and a stimulus $s(x)$ consisting of a drifting sinusoidal grating of frequency $\Omega_{0}$ and modulation 1 , limited by a window of width $w$ centered about the receptive field. The complex function $A \cdot g(x)$ represents a pair of odd and even receptive fields. We assume the measured response $R(w)$ is the modulus of the convolution of stimulus and sensitivity profile, averaged over 1 cycle of drift:

$$
R(w)=\int_{0}^{2 \pi / \Omega_{0}}\left|\int_{-w / 2}^{+w / 2} A \cdot g(x) \cdot s\left(x-x_{0}\right) d x\right| d x_{0} .
$$

Using $s(x)=\frac{1}{2}\left[\exp \left(i \Omega_{0} x\right)-\exp \left(-i \Omega_{0} x\right)\right]$, we obtain

$$
R(w)=B \cdot \int_{-w / 2}^{+w / 2}|g(x)| d x,
$$

with $B=\mathrm{A} \cdot 2 \pi / \Omega_{0}$. Figure 17 shows the corresponding widthresponse function. Its rising slope is $d R /\left.d w\right|_{w=0}=B \cdot|g(0)|=$ $B$, and its asymptote

$$
R_{\infty}=B \cdot \int_{-\infty}^{+\infty} \exp \left[-\frac{x^{2}}{2 \sigma^{2}}\right] d x=B \cdot \sqrt{2 \pi} \cdot \sigma .
$$

If we denote by $w_{1}$ the abscissa of the point of intersection of rising slope and asymptote, we have, from Figure 17,

$$
R_{\infty}=B \cdot w_{1},
$$

and with Equation A3,

$$
w_{1}=\sqrt{2 \pi} \cdot \sigma .
$$

If we express $w_{1}$ in number of grating cycles, $w_{1}=n_{1} \cdot 2 \pi / \Omega_{0}$, we obtain

$$
\Omega_{0}=n_{1} \cdot \sqrt{2 \pi} / \sigma .
$$

Substituting for $\Omega_{0}$ in Equation $A 2$ gives the bandwidth as a function of $n_{1}$,

$$
B_{\mathrm{oct}}=\log _{2}\left[\frac{n_{1} \cdot \sqrt{\pi}+\sqrt{\ln 2}}{n_{1} \cdot \sqrt{\pi}-\sqrt{\ln 2}}\right] .
$$

By solving for $n_{1}$, we obtain also the inverse relationship

$$
n_{1}=(\sqrt{\ln 2 / \pi}) \frac{r+1}{r-1}, \quad \text { with } r=2^{B_{\mathrm{oct}}} .
$$

Equation A4 has been used to calculate the bandwidth of a Gaussian filter whose extent of summation equals that of the median grating cell; Equation A5, to calculate the "complexity index" of grating cells.

Several authors have studied the relationship between the

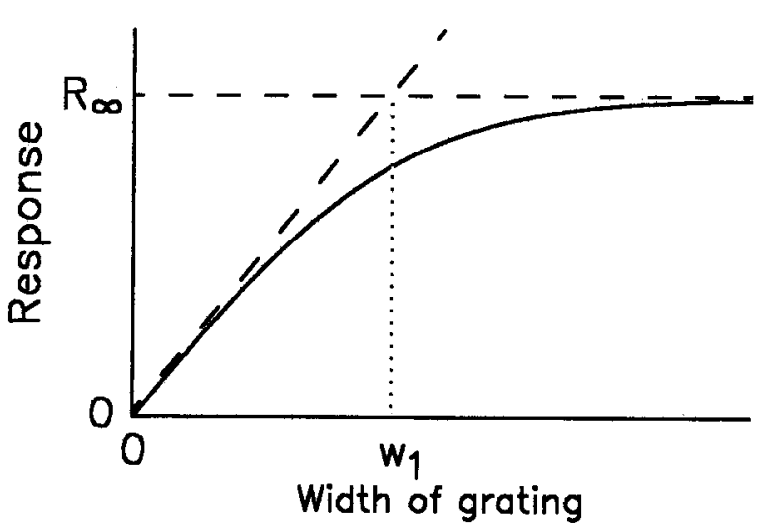

Figure 17. Spatial summation in receptive fields with Gabor-function sensitivity profile. See Appendix for details.

spatial-frequency bandwidth and the number of subregions of receptive fields (cf. von der Heydt, 1987), and Kulikowski et al. (1982) have provided a table of the theoretical values for fields of the Gabor function type. Since the count of subregions depends on the response criterion, values were listed for several criteria. Equation 4 is a more natural way of relating bandwidth to extent of receptive ficld. Since the definition of $n_{1}$ requires only the determination of rising slope and asymptotic level of the width-response function, it does not involve an arbitrary criterion. Also, the slope and asymptote can be estimated even in the presence of a threshold nonlinearity, when part of the subregions are concealed (cf. De Valois et al., 1985).

\section{References}

Albrecht DG, De Valois RL, Thorell LG (1980) Visual cortical neurons: are bars or gratings the optimal stimuli? Science 207:88-90.

Albrecht DG, Farrar SB, Hamilton DB (1984) Spatial contrast adaptation characteristics of neurones recorded in the cat's visual cortex. J Physiol (Lond) 347:713-739.

Andrews BW, Pollen DA (1979) Relationship between spatial frequency selectivity and receptive field profile of simple cells. J Physiol (Lond) 287:163-176.

Blakemore C, Campbell FW (1969) On the existence of neurones in the human visual system selectively sensitive to the orientation and size of retinal images. J Physiol (Lond) 203:237-260.

Campbell FW, Robson JG (1968) Application of Fourier analysis to the visibility of gratings. J Physiol (Lond) 197:551-566.

De Valois RL, De Valois KK (1988) Spatial vision. New York: Oxford UP.

De Valois KK, Tootell RBH (1983) Spatial-frequency-specific inhibition in cat striate cortex cells. J Physiol (Lond) 336:359-376.

De Valois RL, Morgan H, Snodderly DM (1974) Psychophysical studies of monkey vision. III. Spatial luminance contrast sensitivity tests of macaque and human observers. Vision Res 14:75-81.

De Valois KK, De Valois RL, Yund EW (1979) Responses of striate cortex cells to grating and checkerboard patterns. J Physiol (Lond) 291:483-505.

De Valois RL, Albrecht DG, Thorell LG (1982) Spatial frequency selectivity of cells in macaque visual cortex. Vision Res 22:545-559.

De Valois RL, Thorell LG, Albrecht DG (1985) Periodicity of striatecortex-cell receptive fields. J Opt Soc Am A 2:1115-1123.

Dean AF, Tolhurst DJ (1983) On the distinctness of simple and complex cells in the visual cortex of the cat. J Physiol (Lond) 344:305325.

Foster KH, Gaska JP, Nagler M, Pollen DA (1985) Spatial and temporal frequency selectivity of neurones in visual cortical areas $V 1$ and V2 of the macaque monkey. J Physiol (Lond) 365:331-363.

Glezer VD, Ivanoff VA, Tscherbach TA (1973) Investigation of complex and hypercomplex receptive fields of visual cortex of the cat as spatial frequency filters. Vision Res 13:1875-1904.

Glezer VD, Tsherbach TA, Gauselman VE, Bondarko VM (1980) Lin- 
ear and non-linear properties of simple and complex receptive fields in area 17 of the cat visual cortex. Biol Cybern 37:195-208.

Graham N (1977) Visual detection of aperiodic spatial stimuli by probability summation among narrowband channels. Vision Res 17: $637-652$.

Graham N (1985) Detection and identification of near-threshold visual patterns. J Opt Soc Am A 2:1468-1482.

Graham N (1989) Visual pattern analyzers. New York: Oxford UP.

Green DM, Swets JA (1966) Signal detection theory and psychophysics. New York: Wiley.

Hubel DH, Wiesel TN (1962) Receptive fields, binocular interaction and functional architecture in the cat's visual cortex. J Physiol (Lond) 160:106-154.

Hubel DH, Wiesel TN (1968) Receptive fields and functional architecture of monkey striate cortex. J Physiol (Lond) 195:215-243.

Ikeda H, Wright MJ (1975) Spatial and temporal properties of 'sustained' and 'transient' neurons in area 17 of the cat's visual cortex. Exp Brain Res 22:363-383.

Julesz B, Schumer RA (1981) Early visual perception. Annu Rev Psychol 32:575-627.

Kelly DH (1976) Pattern detection and the two-dimensional Fourier transform: flickering checkerboards and chromatic mechanisms. Vision Res 16:277-287.

Kelly DH, Burbeck CA (1984) Critical problems in spatial vision. CRC Crit Rev Biomed Eng 10:125-177.

Kulikowski JJ, Bishop PO (1981) Fourier analysis and spatial representation in the visual cortex. Experientia 37:160-163.

Kulikowski JJ, Marcelja S, Bishop PO (1982) Theory of spatial position and spatial frequency relations in the receptive fields of simple cells in the visual cortex. Biol Cybern 43:187-198.

Lund JS (1973) Organization of neurons in the visual cortex, area 17, of the monkey (Macaca mulatta). J Comp Neurol 147:455-496.

Lund JS, Hendrickson AE, Ogren MP, Tobin EA (1981) Anatomical organization of primate visual cortex VII. J Comp Neurol 202:1945 .

Maffei L, Fiorentini A (1973) The visual cortex as a spatial frequency analyser. Vision Res 13:1255-1267.

Maffei I, Morrone C, Pirchio M, Sandini G (1979) Responses of visual cortical cells to periodic and non-periodic stimuli. J Physiol (Lond) 296:27-47.

Marcelja S (1980) Mathematical description of the responses of simple cortical cells. J Opt Soc Am 70:1297-1300.

McCulloch WS, Pitts W (1943) A logical calculus of ideas immanent in neural nets. Bull Math Biophys 5:115-137.

Morrone MC, Burr DC, Maffei L (1982) Functional implications of cross-orientation inhibition of cortical visual cells. I. Neurophysiological evidence. Proc R Soc Lond [Biol] 216:335-354.

Motter BC, Poggio GF (1984) Binocular fixation in the rhesus monkey: spatial and temporal characteristics. Exp Brain Res 54:304-314.

Movshon JA, Thompson ID, Tolhurst DJ (1978a) Spatial summation in the receptive fields of simple cells in the cat's striate cortex. J Physiol (Lond) 283:53-77.

Movshon JA, Thompson ID, Tolhurst DJ (1978b) Receptive field organization of complex cells in the cat's striate cortex. J Physiol (Lond) 283:79-99.
Nachmias J, Sansbury R, Vassilev A, Weber A (1973) Adaptation to square-wave gratings: in search of the elusive third harmonic. Vision Res 13:1335-1342.

Ohzawa I, Sclar G, Freeman RD (1982) Contrast gain control in the cat visual cortex. Nature 298:266-268.

Olzak LA, Thomas JP (1986) Seeing spatial patterns. In: Handbook of perception and human performance, Vol 1, Sensory processes and perception (Boff KR, Kaufman L, Thomas JP, eds), pp 7.1-7.56. New York: Wiley-Interscience.

Pantle A, Sekuler R (1968) Size-detecting mechanisms in human vision. Science 162:1146-1148.

Poggio GF (1984) Processing of stereoscopic information in primate visual cortex. In: Dynamic aspects of neocortical function (Edelman GM, Gall WE, Cowan WM, eds), pp 613-635. New York: Wiley.

Poggio GF, Doty RW, Talbot WH (1977) Foveal striate cortex of behaving monkey: single neuron responses to square-wave gratings during fixation of gaze. J Neurophysiol 40:1369-1391.

Pollen DA, Ronner SF (1982) Spatial computation performed by simple and complex cells in the visual cortex of the cat. Vision Res 22: 101-118.

Robson JG (1983) Frequency domain visual processing. In: Physical and biological processing of images (Braddick OJ, Sleigh AC, eds), pp 73-87. Berlin: Springer.

Rockel AI, Hiorns RW, Powell TPS (1980) The basic uniformity in structure of the neocortex. Brain 103:221-244.

Schiller PH, Finlay BL, Volman SF (1976) Quantitative studies of single-cell properties in monkey striate cortex. III. Spatial frequency. J Neurophysiol 39:1334-1351.

Schumer RA, Movshon JA (1984) Length summation in simple cells of cat striate cortex. Vision Res 24:565-571.

Shapley R, Lennie P (1985) Spatial frequency analysis in the visual system. Annu Rev Neurosci 8:547-583.

Shapley R, Kaplan E, Soodak R (1981) Spatial summation and contrast sensitivity of $\mathrm{X}$ and $\mathrm{Y}$ cells in the lateral geniculate nucleus of the macaque. Nature 292:543-545.

Spitzer H, Hochstein S (1985) A complex-cell receptive-field model. J Neurophysiol 53:1266-1286.

Stork DG, Levinson IZ, Albrecht DG, De Valois RL, Thorell LG (1982) Receptive fields and the optimal stimulus. Science 216:204-205.

Tolhurst DJ, Movshon JA, Dean AF (1983) The statistical reliability of signals in single neurons in cat and monkey visual cortex. Vision Res 23:775-785.

Van Essen DC, Newsome WT, Maunsell JH (1984) The visual field representation in striate cortex of the macaque monkey: asymmetries, anisotropies, and individual variability. Vision Res 24:429-448.

von der Heydt R (1987) Approaches to visual cortical function. Rev Physiol Biochem Pharmacol 108:69-150.

von der Heydt R, Peterhans E (1989) Mechanisms of contour perception in monkey visual cortex. I. Lines of pattern discontinuity. $\mathrm{J}$ Neurosci 9:1731-1748.

Westheimer G (1984) Spatial vision. Annu Rev Psychol 35:201-226.

Wilson HR, Levi D, Maffei L, Rovamo J, De Valois RL (1989) The perception of form: retina to striate cortex. In: Visual perception: the neurophysiological foundations (Spillman L, Werner JS, eds), pp 231272. New York: Academic. 